\title{
NOVELTIES IN THE ORCHID FLORA OF THE MUNICIPALITY OF BENEDITO NOVO, SANTA CATARINA, BRAZIL, AND AN UPDATED CHECKLIST
}

\author{
Jader Oslim Caetano ${ }^{1}$, Randi Raddatz ${ }^{2}$, Juliane L. Schmitt ${ }^{3}$, Carlos R. Schlemper ${ }^{4}$ \\ \& Leonardo R. S. Guimarães ${ }^{5-6}$ \\ ${ }^{1}$ Rua Holanda, 395, Alto Benedito, Benedito Novo, SC, 89124-000, Brazil \\ ${ }^{2}$ Rua Leopoldo Koprowski, s.n., Alto Benedito, Benedito Novo, SC, 89124-000, Brazil \\ ${ }^{3}$ Rua Manoel Barreto, 54, apto 804, Victor Konder, Blumenau, SC, 89012-134, Brazil \\ ${ }^{4}$ Rua Rio Negrinho, 555, Progresso, Rio do Sul, SC, 89163-640, Brazil \\ ${ }^{5}$ Núcleo de Pesquisa Orquidário do Estado, Instituto de Botânica, Av. Miguel Stéfano, 3687, São Paulo, \\ SP, Caixa Postal 68041, 04045-972, Brazil \\ ${ }^{6}$ Correspondence author: leo.rsguimaraes@hotmail.com
}

\begin{abstract}
In 2013, Caetano and colleagues published two lists of the Orchidaceae of the Municipality of Benedito Novo, Santa Catarina, totalling 99 species. Between January 2014 and September 2016, additional field trips were made and new species were found in the region. Thus, in this paper an updated checklist of the Orchidaceae of Benedito Novo is presented based on herbarium collections and field surveys. A total of 184 species distributed in 66 genera are registered, comprising about $35 \%$ of the species and $60 \%$ of the genera cited for Santa Catarina. The richest genera are Acianthera Scheidw. (18 species), Pabstiella Brieger \& Senghas (14), Epidendrum L., Gomesa R.Br., and Maxillaria Ruiz \& Pav. (13 each). A new combination and a lectotypification in the genus Pabstiella are proposed.
\end{abstract}

KeY worDs: Brazilian Atlantic Forest, flora, Itajaí Valley, monocots, orchids

Introduction. There are few studies with Orchidaceae for the state of Santa Catarina (Favretto \& Geuster 2011, Reis et al. 2011, Siqueira, Zanin \& Menini Neto 2014), and the most important research, conducted between 40 and 60 years ago, focused on the capital Florianopolis and its surroundings (Rohr 1951, Pabst 1951, 1952, 1953, 1954, 1956, 1957, 1959, Klein, Bresolin \& Reis 1977/1978). A vegetation inventory of the Itajaí Valley, including 200 orchid species, was published by Klein (1979). Two lists of 99 species of Orchidaceae were previously prepared as a result of fieldwork carried out between November 2009 and October 2013 in the Municipality of Benedito Novo (Caetano \& Guimarães 2013; Caetano et al. 2013). Expeditions between January 2014 and September 2016 revealed additional new records for the region, which are included in the present, The updated checklist presented here includes those species, as well as numerous nomenclatural emends.

The Brazilian Atlantic Forest is one of the largest tropical forests in the Americas, and its high levels of species richness and endemic taxa renders it one of the 34 global biodiversity hotspots (Myers et al. 2000, Mittermeier et al. 2004). Nevertheless, these forests and species are under intense pressure due to the deforestation suffered in the past.(Myers et al. 2000, Mittermeier et al. 2004). Currently, only about $11 \%$ of the total forest cover is considered to be the original native vegetation (Ribeiro et al. 2009), distributed in small forest fragments. The state of Santa Catarina is fully inserted in the Atlantic Forest biome and currently $23.04 \%$ (about 22,100 km²) remain as total forest remnants of Brazilian Atlantic Forest in Santa Catarina (Fundação SOS Mata Atlântica \& INPE 2011).

Over 20,000 species of vascular plants were estimated for the Brazilian Atlantic Forest (Myers et al. 2000), of which 8,000 (40\%) are endemic to this phytogeographical domain. According to Stehmann et al. (2009), Orchidaceae is one of the most diverse families in the Brazilian Atlantic Forest, represented by more than 1,500 species (BFG 2015). 


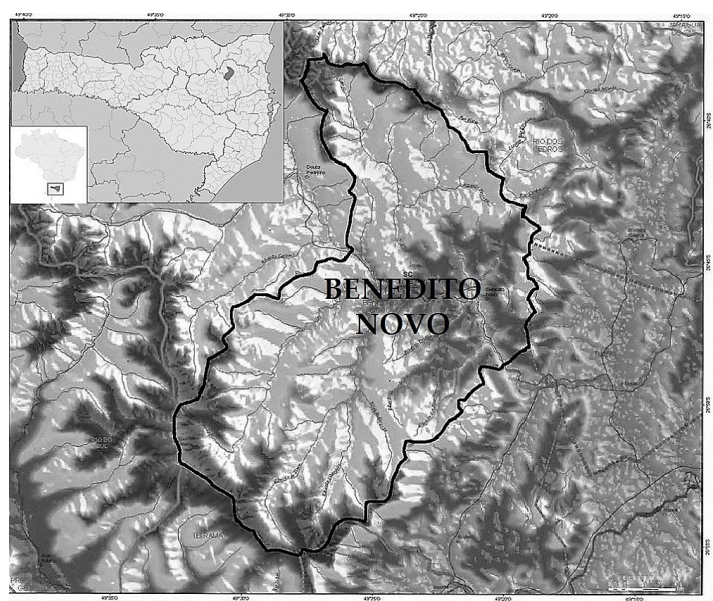

FIgURE 1. Location of the Municipality of Benedito Novo, Santa Catarina, Brazil.
This study aims to present a current inventory of the species of Orchidaceae that occur in the Municipality of Benedito Novo, by including all the most recent findings and the most current taxonomic information. This inventory hopes to contribute to the knowledge of the flora of the Itajaí Valley, and the state of Santa Catarina.

Material and methods. The Municipality of Benedito Novo, with an area of $385,402 \mathrm{~km}^{2}$, is situated in the Itajaí Valley, region of Blumenau, in Eastern state of Santa Catarina (264' $58^{\prime \prime}$ S, 49²1'50" W) (Fig. 1). It has $66.13 \%$ of natural forest, $23.39 \%$ of rural areas, $8.96 \%$ of reforested vegetation, and the urban setting occupies an area of only $1.28 \%$ of the total municipal territory (Fig. 2A). A region well preserved of native forest is the "Campo do Zinco" Farm, where is located the Zinco
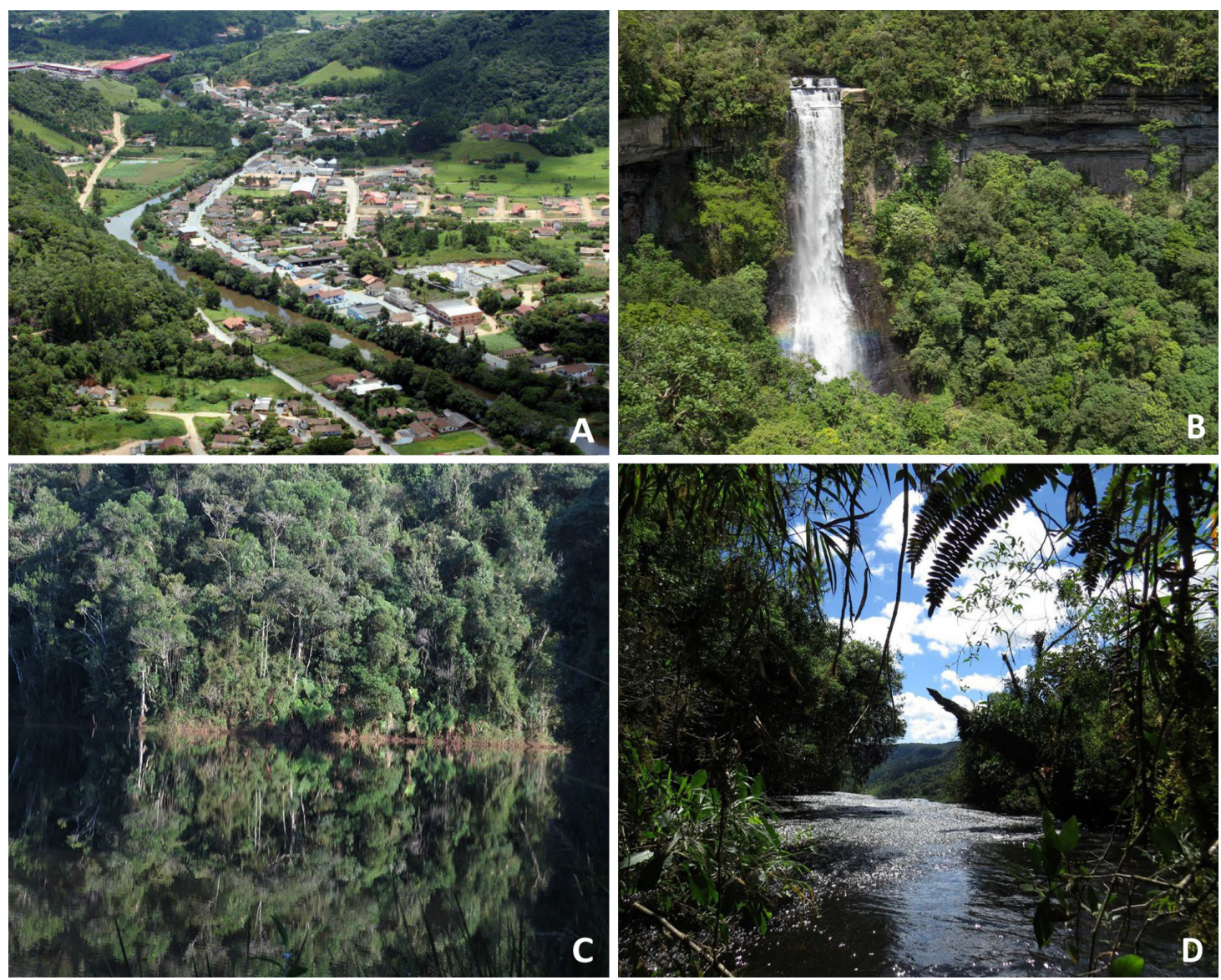

Figure 2. Landscape of the Municipality of Benedito Novo. A. View of the city center of Benedito Novo, showing the Benedito River and areas of Atlantic Forest. B. "Salto do Zinco" Waterfall. C. Riparian forest along an artificial lake in "Campo do Zinco" Farm. D. Zinco River. Photos by Jardel F. de Araujo (A) and Egon Koprowski (B-D). 
River, the "Salto do Zinco" Waterfall (76 m) and many riparian forests (Figs. 2B-D). The climate is oceanic with temperate summer and no dry season (Alvares et al. 2013; type Cfb according to Köppen 1936). The average annual precipitation is $1,561 \mathrm{~mm}$ and the average annual temperature is $20.3^{\circ} \mathrm{C}$ (Climate-Data.org 2016).

The present study is an updated checklist, based on several field surveys made between November 2009 and October 2013 (whose species list were published in Caetano \& Guimarães 2013 and Caetano et al. 2013), plus the excursions carried between January 2014 and September 2016. As in the previous list of 2013, field trips were made mainly at strategic points of the municipality (e.g. riparian forests and top of hills), following the method of active search (for details see Filgueiras et al. 1994). The samples were submitted to the usual taxonomic procedures (Mori et al. 1985) and then deposited in the herbarium of the Universidade Regional de Blumenau (FURB). To update this checklist, we have also reviewed the specimens from the following herbaria: CGMS, FLOR, FUEL, HB, HUCS, ICN, R, RB and UPCB (acronyms according to Thiers 2016).

The circumscription of the genera and species followed BFG (2015) and Flora do Brasil 2020 (2016), except for the subtribes Goodyerinae and Maxillariinae, which are according to Chase et al. (2015). For the subtribe Pleurothallidinae, we followed Flora do Brasil 2020 (2016), but we updated some nomenclatural novelties according to the most recent works, which are indicated in brackets in the Checklist below. Each accepted taxon name is presented in bold with author(s) followed by full citation. If there is a basionym, it is mentioned just after the correct name followed by most popular used homotypic and heterotypic synonym(s) and respective authors (Govaerts et al. 2015). Endemic species to Brazil are indicated with an asterisk $(*)$ and endangered species in category "Vulnerable" are indicated with [VU].

Results. In the Municipality of Benedito Novo, there are 184 species belonging to 66 genera of Orchidaceae. Of these, $160(86.9 \%)$ are epiphytes, $20(10.9 \%)$ terrestrial, and 4 (2.2\%) rupicolous, occurring in several phytophysiognomies. The genus with the highest number of taxa is Acianthera Scheidw. (18 species), followed by Pabstiella Brieger \& Senghas (14), Epidendrum L., Gomesa R.Br., and Maxillaria Ruiz \& Pav. (13 each). In

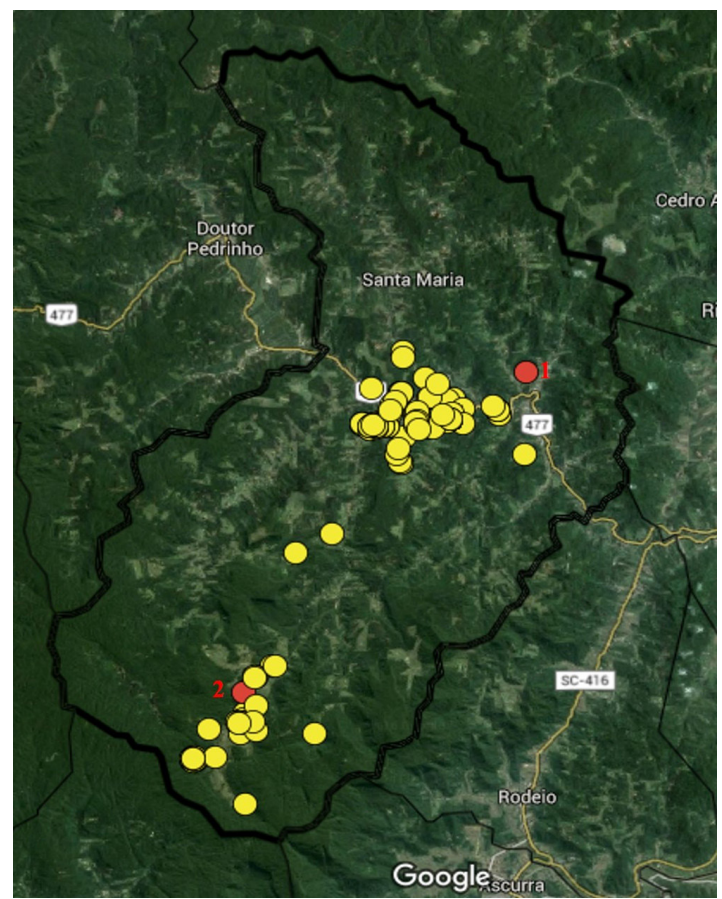

FIGURE 3. Map showing all collection points of Orchidaceae in the Municipality of Benedito Novo. The red dots are: 1: city center of Benedito Novo; 2: "Campo do Zinco" Inn. Mapping: Google Maps.

contrast, 37 genera were represented by a single species. Aside from this, we collected three species not previously referred to the state of Santa Catarina. These species were treated in more detail in Guimarães et al. (2016).

The species were collected mainly in two areas of the municipality. The first along the Benedito River, near the center of the city, the second on the "Campo de Zinco" Farm (Fig. 3).

Eighty-six species recorded for the Municipality of Benedito Novo are endemic to Brazil, and also considered endemic to the Brazilian Atlantic Forest, according to BFG (2015). Among the species found in the area, two are found only in the South Region of Brazil (includes states of Paraná, Santa Catarina and Rio Grande do Sul), viz., Acianthera alborosea (Kraenzl.) Luer and Pabstiella matinhensis (Hoehne) Luer.

Seven species recorded in the Municipality of Benedito Novo are cited as endangered in category "Vulnerable" (VU) by Menini Neto et al. (2013), mainly caused by habitat loss or destruction. They are: Cattleya guttata Lindl., Cattleya intermedia Graham, Dryadella lilliputiana (Cogn.) Luer, Grandiphyllum divaricatum 
(Lind1.) Docha Neto, Grandiphyllum hians (Lind1.) Docha Neto, Grobya fascifera Rchb.f. and Pabstiella carinifera (Barb.Rodr.) Luer.

Discussion. A total of 59 new records of Orchidaceae were added to a previous checklist for the Itajaí Valley elaborated by Klein (1979), which cited a total of 274 species. However, 50 species of Orchidaceae listed in that work are synonyms according to the current nomenclature of Flora do Brasil 2020 (2016); therefore, the number of species of orchids cited for Itajaí Valley is 224, 40 more than was found in Benedito Novo.

This study comprises about $35 \%$ of species and $60 \%$ of the genera cited by Flora do Brasil 2020 (2016) for the state of Santa Catarina. With about $11 \%$ of its original area, the Brazilian Atlantic Forest is currently distributed in scanty fragments that hardly exceed 100 ha (Ranta et al. 1998, Ribeiro et al. 2009). In the state of Santa Catarina, more than $80 \%$ of the remnants of this domain have less than 50 ha (Vibrans et al. 2012). We registered almost 200 species of orchids in ca. 38,540 ha, showing the importance of the conservation of this area.

The main areas of species collected are concentrated along the Benedito River and at the "Campo de Zinco" Farm (Fig. 3). The most probable explanation for this high number of species is that they are preserved areas of the municipality, i.e. the riparian forest of the river and the private property of the farm, which contains a fragment of Atlantic Forest and the collection of specimens is only carried out with prior authorization of the owner. This hypothesis justifies the conservation of the Orchidaceae in the Municipality of Benedito Novo.

Seven species found in Benedito Novo are threatened, mainly due to the loss or destruction of the habitat. Moreover, all of them are included in official red list of threatened species of Brazilian states. Cattleya guttata and $C$. intermedia are classified as "Vulnerable" (VU) in Espírito Santo (Kollmann et al. 2007) and Rio Grande do Sul (Rio Grande do Sul 2002), respectively; and Pabstiella carinifera is treated as "Endangered" (EN) in Paraná (Hatschbach \& Ziller 1995). Dryadella lilliputiana, Grandiphyllum divaricatum, Grobya hians and $G$. fascifera were considered probably extinct (EX) in São Paulo by Mamede et al. (2007); fortunately, we found specimens of these species in Benedito Novo and thus apply conservation actions. In the state of Santa Catarina, no list of threatened plant species was published until now. These facts reinforce the great value of the Municipality of Benedito Novo for the conservation of Orchidaceae.

A comparison of the number of species of Orchidaceae occurring in Benedito Novo with 13 other areas of the Brazilian Atlantic Forest (Table 1), shows that the Municipality of Benedito Novo, Cantareira State Park and Ilha do Cardoso State Park have the highest number of species (184, 159 and 147, respectively), followed by the Fontes do Ipiranga State Park and Serra do Japi (125 each). Among the areas analysed,

TABLE 1. Comparison of the number of genera and species of Orchidaceae among several surveys in areas of Atlantic Forest in Brazil. Locals: $\mathrm{BR}=$ Biological Reserve, $\mathrm{ES}=$ Ecological Station, $\mathrm{MNP}=$ Municipal Nature Park, NP $=\mathrm{National}$ Park, $\mathrm{SP}$ $=$ State Park. States: $\mathrm{ES}=$ Espírito Santo, $\mathrm{MG}=$ Minas Gerais, $\mathrm{PE}=$ Pernambuco, $\mathrm{SC}=$ Santa Catarina, $\mathrm{SP}=\mathrm{São}$ Paulo.

\begin{tabular}{|c|c|c|c|}
\hline Local (State) & $\mathrm{N}^{\circ}$ genera/species & Area (ha) & Reference \\
\hline Fontes do Ipiranga SP (SP) & $52 / 125$ & 345 & Barros 1983 \\
\hline Serra do Brigadeiro SP (MG) & $37 / 55$ & 32,500 & Leoni 1992 \\
\hline Caparaó NP (MG) & $31 / 86$ & 31,853 & Leoni 1997 \\
\hline "Restingas" (ES) & $41 / 71$ & 48,600 & Fraga \& Peixoto 2004 \\
\hline Represa do Grama BR (MG) & $23 / 28$ & 264 & Menini Neto, Almeida \& Forzza 2004 \\
\hline Juréia-Itatins ES (SP) & $40 / 77$ & 84,379 & Catharino \& Barros 2004 \\
\hline Ilha do Cardoso SP (SP) & $66 / 147$ & 22,500 & Romanini \& Barros 2007 \\
\hline Serra do Japi (SP) & $61 / 125$ & 3.54 & Pansarin \& Pansarin 2008 \\
\hline Serras Negra e do Funil (MG) & $50 / 109$ & ca. 10,000 & Abreu, Menini Neto \& Kuono 2011 \\
\hline Francisco Afonso de Melo MNP (SP) & $47 / 67$ & 352.3 & Rodrigues \& Barros 2012 \\
\hline Cantareira SP (SP) & $64 / 159$ & 7,916 & Zandoná \& Catharino 2015 \\
\hline Serra do Urubu (PE) & $50 / 81$ & 982,76 & Pessoa \& Alves 2015 \\
\hline Serra do Tabuleiro SP (SC) & $51 / 92$ & 87,405 & Lenzi et al. 2015 \\
\hline Municipality of Benedito Novo (SC) & $66 / 184$ & 38,540 & This work \\
\hline
\end{tabular}


the Cantareira State Park presents the largest number of species in common with the Municipality of Benedito Novo (76), followed by the Ilha do Cardoso State Park (60). Thus, the Municipality of Benedito Novo is one of the richest areas studied so far, and this high richness of Orchidaceae in the municipality is an important factor to make efforts for its conservation and expansion of biological research and other studies for the area.

The great concentration of species registered here, in the total area of the Municipality of Benedito Novo, is compatible with the number of taxa found in other regions of the Atlantic Forest (Stehmann et al. 2009, BFG 2015). Although Orchidaceae is one of the largest and most important families of flowering plants, the field inventory of this family in the state of Santa Catarina has not been published (Nascimento et al., in prep.). This study contributes to the knowledge of the floristic diversity in the state and highlights the richness of the family, which has great appeal for conservation. Furthermore, it encourages researchers to carry out similar studies in the Municipality of Benedito Novo with other families of embryophytes (bryophytes and vascular plants).

\section{H E C K L I S T}

\section{ACianthera Schweid.}

Acianthera alborosea (Kraenzl.) Luer, Monogr. Syst. Bot. Missouri Bot. Gard. 95: 253. 2004 (Fig. 4).* Octomeria alborosea Kraenzl., Ark. Bot. 16(8): 16. 1921.

Pleurothallis alborosea (Kraenzl.) Brade, Rodriguésia 1(2): 47. 1935.

HABIT AND HABITAT: Epiphyte; forest interior. VOUCHER: Schmitt et al. 1801 (FURB).

Acianthera aphthosa (Lindl.) Pridgeon \& M.W.Chase, Lindleyana 16(4): 242. 2001 (Fig. 4)

Pleurothallis aphthosa Lindl., Edwards's Bot. Reg. 24(Misc.): 42.1838.

Specklinia aphthosa (Lindl.) F.Barros, Hoehnea 10: 109. 1984.

HABIT AND HABITAT: Epiphyte; riparian forest.

VOUCher: Caetano 190 (spirit, FURB).

Acianthera auriculata (Lindl.) Pridgeon \& M.W.Chase, Lindleyana 16(4): 242. 2001.

Pleurothallis auriculata Lindl., Companion Bot. Mag. 2: 356. 1837.

Humboltia auriculata (Lindl.) Kuntze, Revis. Gen. P1. 2: 667. 1891.

Specklinia auriculata (Lindl.) F.Barros, Hoehnea 10: 109. 1984.

Arthrosia auriculata (Lindl.) Luer, Monogr. Syst. Bot. Missouri Bot. Gard. 105: 248. 2006.

HABIT AND HABITAT: Epiphyte; riparian forest. Voucher: Caetano 12 (FURB).

Acianthera bragae (Ruschi) F.Barros, Hoehnea 30(3): 183. 2003 (Fig. 4).*

Physosiphon bragae Ruschi, Bol. Mus. Biol. Prof. Mello-Leitão, Sér. Bot. 27: 1. 1970.
Physosiphon pubescens Barb.Rodr., Gen. Spec. Orchid. 1: 27. 1877.

Phloeophila pubescens (Barb.Rodr.) Garay, Orquideología 9(2): 118. 1974.

Sarracenella pubescens (Barb.Rodr.) Luer, Selbyana 5(3-4): 388. 1981.

Pleurothallis sarracenia Luer, Monogr. Syst. Bot. Missouri Bot. Gard. 20: 73. 1986.

Acianthera sarracenia (Luer) Pridgeon \& M.W.Chase, Lindleyana 16(4): 246. 2001.

Habit AND HABITAT: Epiphyte; riparian forest.

VOUCHER: Caetano s.n. (FURB 36259).

Acianthera calopedilon Toscano \& Luer, Lankesteriana 15(1): 79.2015 (Fig. 4).

HABIT AND HABITAT: Epiphyte; riparian forest.

Voucher: Caetano 31 (FURB).

Acianthera crepiniana (Cogn.) Chiron \& Van den Berg, Richardiana 12(2): 73. 2012.*

Pleurothallis crepiniana Cogn. in Mart., Fl. Bras. 3(4): 542. 1896.

Pleurobotryum crepinianum (Cogn.) Hoehne, Bol. Mus. Nac. Rio de Janeiro 12(2): 28. 1936.

HaBIT AND HABITAT: Epiphyte; riparian forest.

Voucher: Caetano s.n. (FURB 37348).

Acianthera exarticulata (Barb.Rodr.) Pridgeon \& M.W.Chase, Lindleyana 16(4): 243. 2001.*

Pleurothallis exarticulata Barb.Rodr., Gen. Spec. Orchid. 2: 27. 1881.

HABIT AND HABITAT: Epiphyte; riparian forest.

Voucher: Caetano 185 (spirit, FURB).

Acianthera glanduligera (Lindl.) Luer, Monogr. Syst. Bot. Missouri Bot. Gard. 95: 253. 2004.*

Pleurothallis glanduligera Lindl., Companion Bot. Mag. 2: 355. 1837. 
Humboltia glanduligera (Lindl.) Kuntze, Revis. Gen. P1. 2: 667. 1891.

Pleurothallis cryptoceras Rchb.f., Flora 69: 554. 1886.

Acianthera cryptoceras (Rchb.f.) F.Barros, Hoehnea 30(3): 185. 2003.

Pleurothallis cearensis Schltr., Repert. Spec. Nov. Regni Veg. 17: 271. 1921.

Acianthera cearensis (Schltr.) Pridgeon \& M.W.Chase, Lindleyana 16(4): 242. 2001.

Pleurothallis iguapensis Schltr., Anexos Mem. Inst. Butantan, Secç. Bot. 1(4): 48. 1922.

Anathallis iguapensis (Schltr.) Pridgeon \& M.W.Chase, Lindleyana 16(4): 249. 2001.

Acianthera iguapensis (Schltr.) F.Barros, Orchid Memories: 10. 2004.

HABIT AND HABITAT: Epiphyte; anthropic area (pasture).

Voucher: Caetano s.n. (FURB 36290).

Acianthera hygrophila (Barb.Rodr.) Pridgeon \& M.W.Chase, Lindleyana 16(4): 244. 2001.

Pleurothallis hygrophila Barb.Rodr., Gen. Spec. Orchid. 1: 7. 1877.

Specklinia hygrophila (Barb.Rodr.) F.Barros, Hoehnea 10: 110. 1984.

Stelis hygrophila (Barb.Rodr.) Pridgeon \& M.W.Chase, Lindleyana 16(4): 263. 2001.

Arthrosia hygrophila (Barb.Rodr.) Luer, Monogr. Syst. Bot. Missouri Bot. Gard. 105: 249. 2006.

Pleurothallis barbacenensis Barb.Rodr., Gen. Spec. Orchid. 2: 11. 1881.

Acianthera barbacenensis (Barb.Rodr.) Pridgeon \& M.W.Chase, Lindleyana 16(4): 242. 2001.

Specklinia barbacenensis (Barb.Rodr.) Luer, Monogr. Syst. Bot. Missouri Bot. Gard. 95: 259. 2004. Arthrosia barbacenensis (Barb.Rodr.) Luer, Monogr. Syst. Bot. Missouri Bot. Gard. 105: 248. 2006.

Pleurothallis platysemos Rchb.f., Flora 69: 555. 1886.

HABIT AND HABITAT: Epiphyte; forest interior.

Vouchers: Caetano s.n. (FURB 36286); Schmitt et al. 499 (FURB).

Acianthera luteola (Lindl.) Pridgeon \& M.W.Chase, Lindleyana 16(4): 244. 2001 (Fig. 4).*

Pleurothallis luteola Lindl., Edwards's Bot. Reg. 27(Misc.): 1. 1841.

Specklinia luteola (Lindl.) F.Barros, Hoehnea 10:
110. 1984.

HABIT AND HABITAT: Epiphyte; forest interior.

Vouchers: Klein \& Bresolin 10891 (FLOR); Caetano s.n. (FURB 37779).

Acianthera macropoda (Barb.Rodr.) Pridgeon \& M.W.Chase, Lindleyana 16(4): 244. 2001.*

Pleurothallis macropoda Barb.Rodr., Gen. Spec. Orchid. 2: 25. 1881.

HABIT AND HABITAT: Epiphyte; anthropic area (pasture).

VOUCHER: Caetano 192 (spirit, FURB).

Acianthera nemorosa (Barb.Rodr.) F.Barros, Hoehnea 30(3): 186. 2003.*

Pleurothallis nemorosa Barb.Rodr., Gen. Spec. Orchid. 1: 11. 1877.

Pleurothallis farinosa Pabst, Arch. Jard. Bot. Rio de Janeiro 14: 10. 1956.

Acianthera farinosa (Pabst) Luer, Monogr. Syst. Bot. Missouri Bot. Gard. 95: 253. 2004.

HABIT AND HABITAT: Epiphyte; riparian forest.

Voucher: Caetano 59 (FURB).

Acianthera oligantha (Barb.Rodr.) F.Barros, Hoehnea 30(3): 186. 2003.*

Pleurothallis oligantha Barb.Rodr., Gen. Spec. Orchid. 2: 33. 1881.

HABIT AND HABITAT: Epiphyte; forest interior.

Voucher: Caetano s.n. (FURB 36260).

Acianthera pubescens (Lindl.) Pridgeon \& M.W.Chase, Lindleyana 16(4): 245. 2001 (Fig. 4). Pleurothallis pubescens Lindl., Companion Bot. Mag. 2(24): 355. 1837.

Humboltia pubescens (Lindl.) Kuntze, Revis. Gen. P1. 2: 668. 1891.

Pleurothallis smithiana Lindl., Edwards's Bot. Reg. 29(Misc.): 57. 1843.

Humboltia smithiana (Lindl.) Kuntze, Revis. Gen. P1. 2: 668. 1891.

Pleurothallis riograndensis Barb.Rodr., Gen. Spec. Orchid. 2: 28. 1881.

HABIT AND HABITAT: Epiphyte; forest interior, riparian forest.

Vouchers: Caetano 188 (spirit, FURB); Caetano 218 (spirit, FURB).

Acianthera punctatiflora (Luer) Pridgeon \& M.W.Chase, Lindleyana 16(4): 246. 2001 (Fig. 4).* Pleurothallis punctatiflora Luer, Monogr. Syst. Bot. Missouri Bot. Gard. 20: 16. 1986. 
Cryptophoranthus punctatus Barb.Rodr., Rev. Engenh. 3: 9. 1881; Gen. Spec. Orchid. 2: 80. 1881. HABIT AND HАВITAT: Epiphyte; forest interior (riparian forest).

Voucher: Caetano s.n. (FURB 38093).

Acianthera saundersiana (Rchb.f.) Pridgeon \& M.W.Chase, Lindleyana 16(4): 246. 2001 (Fig. 4). Pleurothallis saundersiana Rchb.f., Gard. Chron. 1866: 74. 1866.

Specklinia saundersiana (Rchb.f.) F.Barros, Hoehnea 10: 110. 1984.

Pleurothallis butantanensis Hoehne \& Schltr., Arch. Bot. São Paulo 1: 209. 1926.

HABIT AND HABITAT: Epiphyte; forest interior.

Voucher: Caetano \& Raddatz 69 (FURB).

Acianthera saurocephala (G.Lodd.) Pridgeon \& M.W.Chase, Lindleyana 16(4): 246. 2001 (Fig. 4).* Pleurothallis saurocephala G.Lodd., Bot. Cab. 16: t. 1571.1830 .

Humboltia saurocephala (G.Lodd.) Kuntze, Revis. Gen. P1. 2: 668. 1891.

HABIT AND HABITAT: Epiphyte; anthropic area (pasture).

Voucher: Caetano 56 (FURB).

Acianthera sonderiana (Rchb.f.) Pridgeon \& M.W.Chase, Lindleyana 16(4): 246. 2001.

Pleurothallis sonderiana Rchb.f., Linnaea 22: 830. 1850.

Humboltia sonderiana (Rchb.f.) Kuntze, Revis. Gen. P1. 2: 668. 1891.

Specklinia sonderiana (Rchb.f.) F.Barros, Hoehnea 10: 110.1984.

HABIT AND HABITAT: Epiphyte; forest interior.

Vouchers: Caetano s.n. (FURB 40577); Schmitt et al. 1738 (FURB); Korte \& Kniess 2079 (FURB).

Anathallis Barb.Rodr.

Anathallis heterophylla Barb.Rodr., Gen. Spec. Orchid. 2: 74. 1881. [Toscano de Brito \& Luer 2015] (Fig. 4)*

Pleurothallis heterophylla (Barb.Rodr.) Cogn. in Mart., F1. Bras. 3(4): 556. 1896.

Specklinia heterophylla (Barb.Rodr.) Luer, Monogr. Syst. Bot. Missouri Bot. Gard. 95: 261. 2004.

Pleurothallis liparanges Rchb.f., Gard. Chron. n.s. 23: 532.1885.
Specklinia liparanges (Rchb.f.) Luer, Monogr. Syst. Bot. Missouri Bot. Gard. 95: 261. 2004.

Anathallis liparanges (Rchb.f.) Luer, Novon 18(1): 78. 2008.

HABIT AND HABITAT: Epiphyte; anthropic area (urban area), forest interior.

Voucher: Caetano \& Almeida 199 (spirit, FURB); Caetano 211 (spirit, FURB).

Anathallis linearifolia (Cogn.) Pridgeon \& M.W.Chase, Lindleyana 16(4): 249. 2001 (Fig. 4). Pleurothallis linearifolia Cogn. in Mart., F1. Bras. 3(4): 573. 1896.

Specklinia linearifolia (Cogn.) Luer, Monogr. Syst. Bot. Missouri Bot. Gard. 95: 261. 2004.

Pleurothallis depauperata Cogn. in Mart., Fl. Bras. 3(4): 574. 1896.

Specklinia depauperata (Cogn.) F.Barros, Hoehnea 10: 109. 1984.

Pleurothallis glossochila Kraenzl., Ark. Bot. 16(8): 14. 1921.

HABIT AND HABITAT: Epiphyte; riparian forest.

Vouchers: Bresolin 664 (FLOR); Caetano 51 (FURB).

Anathallis obovata (Lindl.) Pridgeon \& M.W.Chase, Lindleyana 16(4): 250. 2001 (Fig. 4).

Specklinia obovata Lindl., Edwards's Bot. Reg. 25(Misc.): 86. 1839.

Pleurothallis obovata (Lindl.) Lindl., Edwards's Bot. Reg. 28(Misc.): 75. 1842.

Pleurothallis minutiflora Cogn. in Mart., Fl. Bras. 3(4): 564. 1896, nom. illeg.

Pleurothallis modestiflora Schltr., Repert. Spec. Nov. Regni Veg. Beih. 35: 56. 1925.

HABIT AND HABITAT: Epiphyte; riparian forest.

Voucher: Caetano 77 (FURB).

\section{Aspidogyne Garay}

Aspidogyne rosea (Lindl.) Meneguzzo, Orquidário 26(3): 90. 2012.

Physurus roseus Lindl., Gen. Sp. Orchid. P1.: 501. 1840.

Microchilus roseus (Lind1.) D.Dietr., Syn. P1. 5: 166. 1852.

Erythrodes rosea (Lindl.) Ames, Orchidaceae 7: 75. 1922.

Ligeophila rosea (Lindl.) Garay, Bradea 2(28): 195. 1977. 
HABIT AND HABITAT: Terrestrial; forest edge.

Voucher: Caetano 76 (FURB).

Aspidogyne schlechteriana (Hoehne) Meneguzzo, Orquidário 26(3): 90. 2012.*

Physurus schlechterianus Hoehne, Fl. Brasilica 12(2): 354. 1945.

Erythrodes schlechteriana (Hoehne) Pabst,

Sellowia 7: 176. 1956.

Platythelys schlechteriana (Hoehne) Garay, Bradea 2(28): 198. 1977.

HABIT AND HABITAT: Terrestrial; forest interior.

Voucher: Caetano et al. 47 (FURB).

\section{Barbosella Schltr.}

Barbosella cogniauxiana (Speg. \& Kraenzl.) Schltr., Repert. Spec. Nov. Regni Veg. 15: 260. 1918.

Restrepia cogniauxiana Speg. \& Kraenzl., Orchis 2: 127. 1908 (Fig. 4).

Barbosella porschii (Kraenzl.) Schltr., Repert. Spec. Nov. Regni Veg. 15: 263. 1918.

Restrepia porschii Kraenzl., Kongl. Svenska Vetensk. Acad. Handl., n.s. 46(10): 51. 1911.

Barbosella handroi Hoehne, Bol. Agric. (São Paulo) 34: 613.1934 ("1933").

HABIT AND HABITAT: Epiphyte; riparian forest.

Voucher: Caetano s.n. (FURB 38277).

Barbosella gardneri (Lindl.) Schltr., Repert. Spec. Nov. Regni Veg. 15: 261. 1918 (Fig. 4).*

Pleurothallis gardneri Lindl., Edwards's Bot. Reg. 28(Misc.): 83. 1842.

Restrepia gardneri (Lindl.) Benth. in Benth. \& Hook., Gen. Pl. 3(2): 491. 1883.

Humboltia gardneri (Lindl.) Kuntze, Revis. Gen. Pl. 2: 667. 1891.

HABIT AND HABITAT: Epiphyte; forest edge.

VOUCher: Caetano s.n. (FURB 37329).

Barbosella miersii (Lindl.) Schltr., Repert. Spec. Nov. Regni Veg. 15: 262. 1918.*

Pleurothallis miersii Lindl., Edwards's Bot. Reg. 28(Misc.): 84. 1842.

Restrepia miersii (Lindl.) Rchb.f. in Wawra, Bot. Ergebn.: 150. 1866.

Humboltia miersii (Lindl.) Kuntze, Revis. Gen. Pl. 2: 668. 1891.

Barbrodria miersii (Lindl.) Luer, Selbyana 5(3-4): 386. 1981.
HABIT AND HABITAT: Epiphyte; riparian forest, forest interior.

Vouchers: Caetano s.n. (FURB 37334); Schmitt et al. 1749 (FURB).

\section{BIFRENARIA Lindl.}

Bifrenaria aureofulva (Hook.) Lindl., Edwards's Bot. Reg. 29(Misc.): 52.1843 (Fig. 4).*

Maxillaria aureofulva Hook., Bot. Mag. 65: t. 3629. 1838.

Stenocoryne aureofulva (Hook.) Kraenzl. in Rchb.f.. Xenia Orchid. 3: 142. 1896.

Epidendrum secundum Vell., Fl. Flumin. Icon. 9: t. 9. 1831 (“1827”), nom. illeg.

Stenocoryne secunda (Vell.) Hoehne, Arq. Bot. Estado São Paulo n.s. 2(1): 13. 1944.

Bifrenaria secunda (Vell.) Pabst, Orquídea (Rio de Janeiro) 29(4): 165. 1967.

HABIT AND HABITAT: Epiphyte; forest edge, forest interior.

VOUCHERS: Klein \& Bresolin 10890 (FLOR); Caetano s.n. (FURB 37346); Schmitt et al. 1809 (FURB).

Bifrenaria harrisoniae (Hook.) Rchb.f., Bonplandia 3: 217.1855 (Fig. 4).*

Dendrobium harrisoniae Hook., Exot. Fl. 2(14): t. 120. 1824.

Maxillaria harrisoniae (Hook.) Lindl., Bot. Reg. 11: t. 897. 1825.

Colax harrisoniae (Hook.) Lindl. ex Spreng., Syst. Veg. (ed. 16) 3: 727. 1826.

Stanhopea harrisoniae (Hook.) P.N.Don in Donn, Hortus Cantabrig., ed. 13: 607. 1845.

Lycaste harrisoniae (Hook.) G.Don ex Loudon, Encycl. Pl. (new ed.) 2: 1468. 1855.

HABIT AND HABITAT: Epiphyte; forest interior, riparian forest.

Vouchers: Caetano s.n. (FURB 39662); Schmitt et al. 524 (FURB).

Bifrenaria inodora Lindl., Edwards's Bot. Reg. 29(Misc.): 48.1843 (Fig. 5).*

Stenocoryne inodora (Lindl.) Kraenzl. in Rchb.f.. Xenia Orchid. 3: 142. 1896.

HABIT AND HABITAT: Epiphyte; anthropic area (pasture).

Voucher: Caetano s.n. (FURB 36933). 

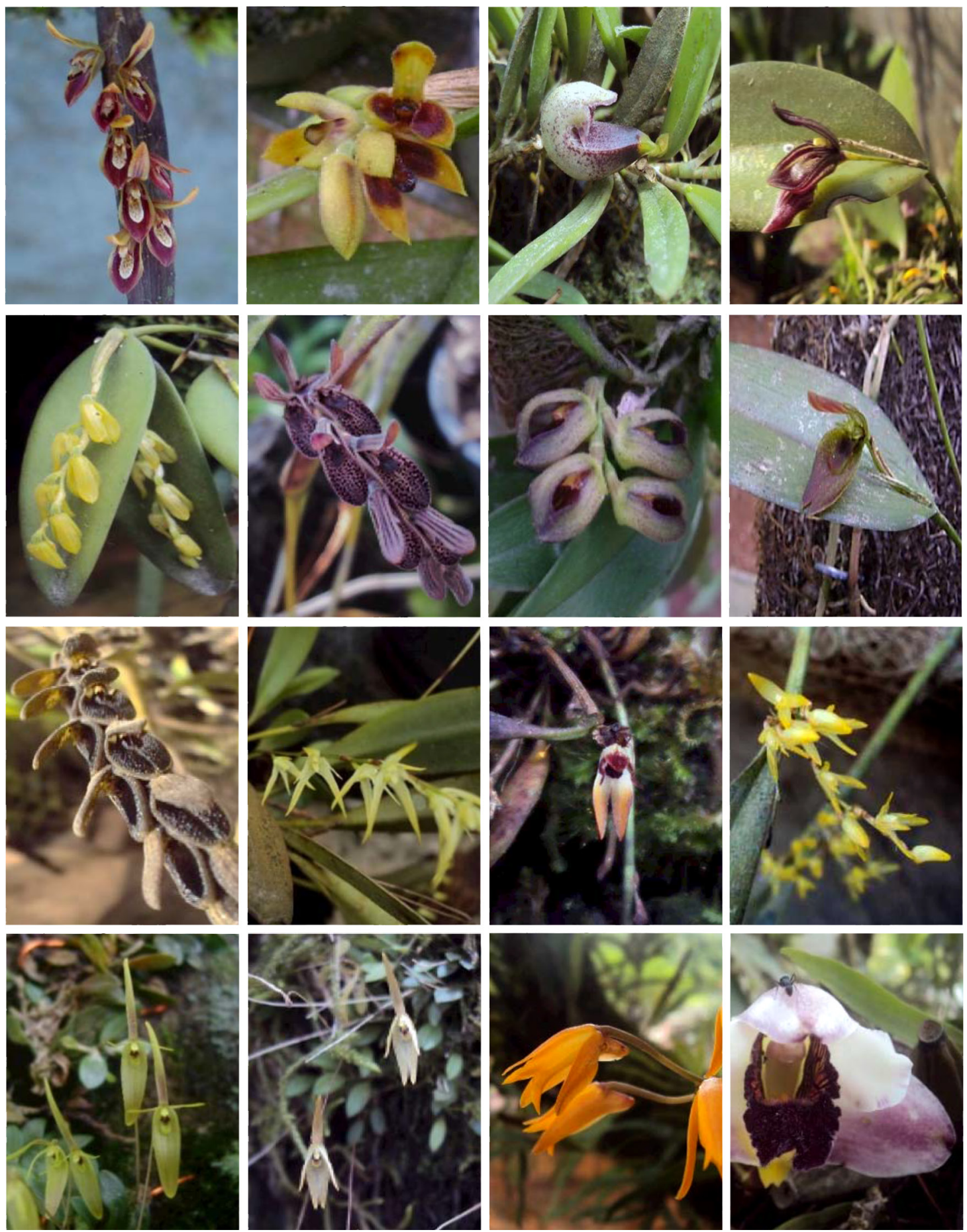

Figure 4. From top left to bottom right: Acianthera alborosea. A. aphtosa. A. bragae. A. calopedilon. A. luteola. A. pubescens. A. punctatiflora. A. saundersiana. A. saurocephala. Anathallis heterophylla. A. linearifolia. A. obovata. Barbosella cogniauxiana. B. gardneri. Bifrenaria aureofulva. B. harrisoniae. All courtesy of The Field Museum, Chicago. 

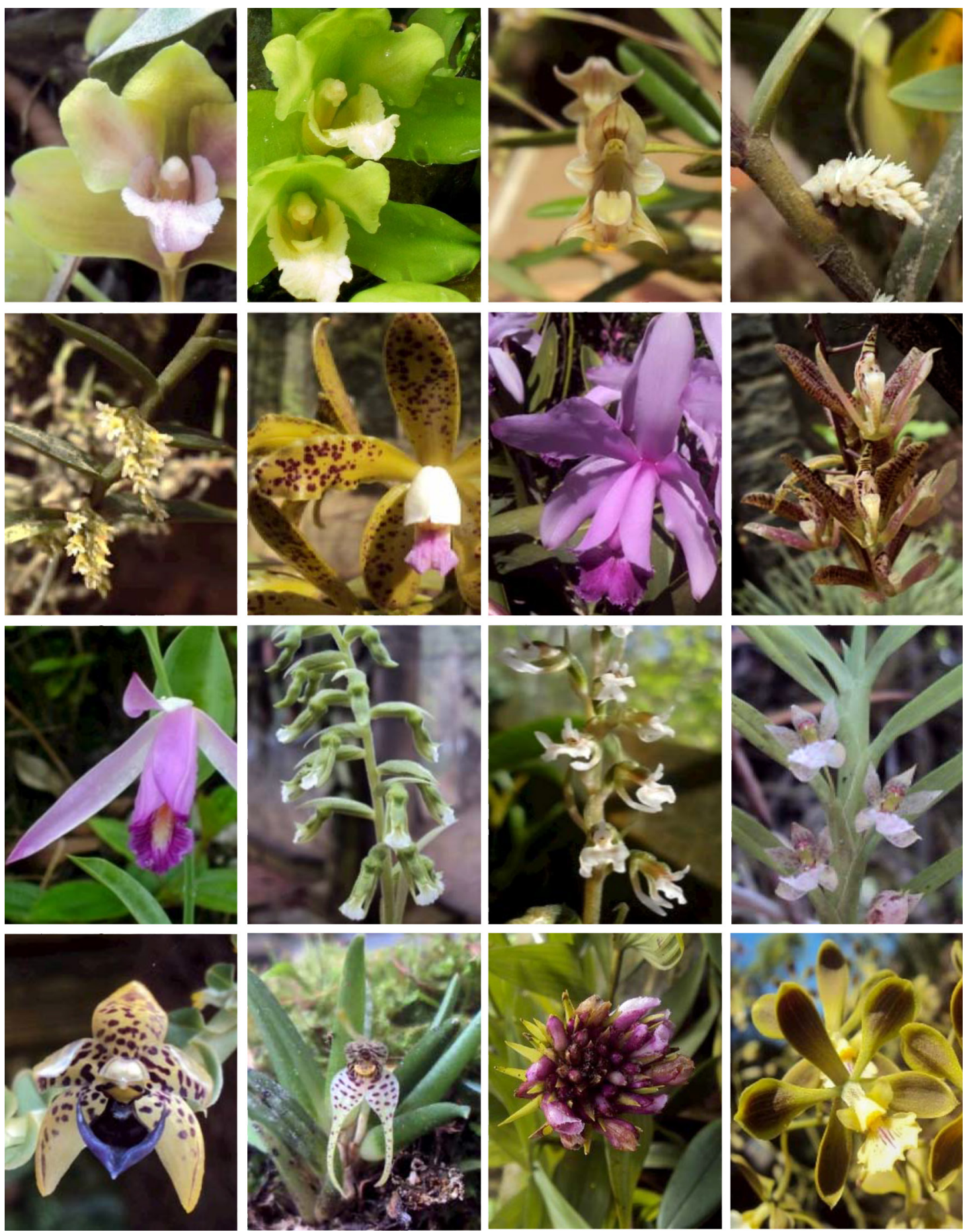

FIgURE 5. From top left to bottom right: Bifrenaria inodora. B. inodora var. alba. Bulbophyllum napellii. Campylocentrum densiflorum. C. pauloense. Cattleya guttata. C. intermedia. Cirrhaea dependens. Cleistes libonii. Cyclopogon congestus. C. subalpestris. Dichaea cogniauxiana. D. pendula. Dryadella lilliputiana. Elleanthus brasiliensis. Encyclia patens var. patens. All courtesy of The Field Museum, Chicago. 
Bulbophyllum Thouars

Bulbophyllum glutinosum (Barb.Rodr.) Cogn. in Mart., Fl. Bras. 3(5): 597. 1902.*

Didactyle glutinosa Barb.Rodr., Gen. Spec. Orchid. 2: 126. 1881.

HABIT AND HABITAT: Epiphyte; forest edge.

Voucher: Caetano s.n. (FURB 38756).

Bulbophyllum granulosum Barb.Rodr., Gen. Spec. Orchid. 1: 41. 1877.*

Didactyle granulosa (Barb.Rodr.) Barb.Rodr., Gen. Spec. Orchid. 2: 125. 1881.

HABIT AND HABITAT: Epiphyte; anthropic area (pasture).

Voucher: Caetano \& Schlemper 204 (spirit, FURB).

Bulbophyllum napellii Lindl., Ann. Mag. Nat. Hist. 10: 185. 1842 (Fig. 5).

Phyllorkis napelli (Lindl.) Kuntze, Revis. Gen. P1. 2: 677. 1891.

HABIT AND HABITAT: Epiphyte; forest interior.

Vouchers: Bresolin 663 (FLOR, HB); Schmitt et al. 756 (FURB); Schmitt et al. 1807 (FURB).

Bulbophyllum regnellii Rchb.f., Linnaea 22: 835. 1850.

Didactyle regnellii (Rchb.f.) Barb.Rodr., Gen. Spec. Orchid. 2: 123. 1881.

HABIT AND HABITAT: Epiphyte; forest edge.

VOUCher: Caetano et al. 50 (FURB).

\section{Campylocentrum Benth.}

Campylocentrum densiflorum Cogn. in Mart., Fl. Bras. 3(6): 511. 1906 (Fig. 5).

HABIT AND HABITAT: Epiphyte; riparian forest.

VOUCHER: Caetano et al. 49 (FURB).

Campylocentrum grisebachii Cogn. in Mart., Fl. Bras. 3(6): 522. 1906.

Campylocentrum burchellii Cogn. in Mart., Fl. Bras. 3(6): 522. 1906

Habit AND habitat: Epiphyte; anthropic area (pasture).

Voucher: Caetano 194 (spirit, FURB).

Campylocentrum ornithorrhynchum (Lind1.) Rolfe, Orchid Rev. 11: 246. 1903.*

Angraecum ornithorrhynchum Lindl., Edwards's Bot. Reg. 26: t. 68. 1840.

Aeranthes ornithorrhyncha (Lindl.) Rchb.f. in Walp., Ann. Bot. Syst. 6(6): 903. 1864.
HaBIT AND HABITAT: Epiphyte; anthropic area (reforested).

Voucher: Caetano 221 (spirit, FURB).

Campylocentrum parahybunense (Barb.Rodr.) Rolfe, Orchid Rev. 11: 246. 1903.*

Aeranthes parahybunensis Barb.Rodr., Gen. Spec. Orchid. 2: 245. 1882.

HABIT AND HABITAT: Epiphyte; riparian forest.

Voucher: Caetano s.n. (FURB 40574).

Campylocentrum pauloense Hoehne \& Schltr., Arch. Bot. São Paulo 1: 297. 1926 (Fig. 5).

HABIT AND HABITAT: Epiphyte; forest edge.

Voucher: Caetano 48 (FURB).

Campylocentrum ulaei Cogn. in Mart., Fl. Bras. 3(6): 514. 1906

HABIT AND HABITAT: Epiphyte; riparian forest.

Voucher: Caetano et al. 200 (spirit, FURB).

Capanemia Barb.Rodr.

Capanemia gehrtii Hoehne, Arq. Bot. Estado São Paulo, n.s. 1: 43. 1939.*

HABIT AND HABITAT: Epiphyte; anthropic area (urban area).

VOUCher: Caetano s.n. (FURB 36261).

Capanemia thereziae Barb.Rodr., Gen. Spec. Orchid. 2: 244. 1882.*

Quekettia thereziae (Barb.Rodr.) Cogn. in Mart., Fl. Bras. 3(6): 200. 1905.

HABIT AND HABITAT: Epiphyte; anthropic area (urban area).

Voucher: Caetano 213 (spirit, FURB).

Cattleya Lindl.

Cattleya guttata Lindl., Edwards's Bot. Reg. 17: t. 1406. 1831 (Fig. 5).* [VU]

Cattleya elatior Lindl., Gen. Sp. Orchid. Pl.: 117. 1833.

HABIT AND HABITAT: Rupicolous; riparian forest.

Voucher: Caetano 64 (FURB).

Cattleya intermedia Graham, Bot. Mag. 55: t. 2851. 1828 (Fig. 5). [VU]

HABIT AND HABITAT: Epiphyte; riparian forest.

Voucher: Caetano 78 (FURB).

Cirrhaea Lindl.

Cirrhaea dependens (G.Lodd.) Loudon, Hort. Brit.: 370. 1830 (Fig. 5).* 
Cymbidium dependens G.Lodd., Bot. Cab. 10(4): t. 936.1825.

HABIT AND HABITAT: Epiphyte; anthropic (urban) area. Voucher: Caetano s.n. (FURB 40573).

Cleistes Rich. ex Lindl.

Cleistes libonii (Rchb.f.) Schltr., Arch. Bot. São Paulo 1: 179. 1926 (Fig. 5).*

Pogonia libonii Rchb.f., Xenia Orchid. 2: 91. 1865. Pogonia macrantha Barb.Rodr., Rev. Engenh. 3: 144. 1881.

Cleistes macrantha (Barb.Rodr.) Schltr., Arch. Bot. São Paulo 1: 179. 1926.

Pogonia revoluta Barb.Rodr., Rev. Engenh. 3: 144. 1881.

Cleistes revoluta (Barb.Rodr.) Schltr., Repert. Spec. Nov. Regni Veg. Beih. 35: 26. 1925.

Pogonia magnifica Schltr., Repert. Spec. Nov. Regni Veg. 16: 316. 1920.

Cleistes magnifica (Schltr.) Schltr., Arch. Bot. São Paulo 1: 180. 1926.

Habit and habitat: Terrestrial; anthropic area (urban area).

Vouchers: Costa s.n. (R 198377); Caetano s.n. (FURB 38279, HUCS 39284).

\section{CORYMBorkis Thouars}

Corymborkis flava (Sw.) Kuntze, Revis. Gen. Pl. 2: 658. 1891.

Serapias flava Sw., Prodr.: 119. 1788.

HABIT AND HABITAT: Terrestrial; forest interior.

VOUCHER: Caetano 7 (FURB).

\section{Cranichis Sw.}

Cranichis candida (Barb.Rodr.) Cogn. in Mart., Fl. Bras. 3(4): 248. 1895.

Cystochilum candidum Barb.Rodr., Gen. Spec. Orchid. 1: 198. 1877.

Cranichis similis Rchb.f., Otia Bot. Hamburg. 2: 83. 1881.

HABit AND HABitAT: Terrestrial; anthropic area (urban area).

Voucher: Caetano 21 (FURB).

\section{Cyclopogon C.Pres1}

Cyclopogon congestus (Vell.) Hoehne, Fl. Brasilica 12(2): 209. 1945 (Fig. 5).
Serapias congesta Vell., Fl. Flumin. Icon. 9: t. 54. 1831.

Beadlea congesta (Vell.) Garay, Bot. Mus. Leafl. 28(4): 300. 1982 (“1980”).

Cyclopogon alpestris var. bidentatus Barb.Rodr., Gen. Spec. Orchid. 2: 283. 1882.

Cyclopogon bidentatus (Barb.Rodr.), Szlach., Candollea 48(2): 435. 1993.

Beadlea bidentata (Barb.Rodr.) Garay, Bot. Mus. Leafl. 28(4): 299. 1982 (“1980”).

HABIT AND HABITAT: Epiphyte; forest edge.

Voucher: Caetano 33 (FURB).

Cyclopogon iguapensis Schltr., Anexos Mem. Inst. Butantan, Secç. Bot. 1(4): 25. 1922.*

Beadlea iguapensis (Schltr.) Garay, Bot. Mus. Leafl. 28(4): 300. 1982 (“1980”).

HABIT AND HABITAT: Epiphyte; forest interior.

Voucher: Caetano 27 (FURB).

Cyclopogon longibracteatus (Barb.Rodr.) Schltr., Beih. Bot. Centralbl. 37(2): 390. 1920.

Spiranthes longibracteata Barb.Rodr., Gen. Spec. Orchid. 1: 185. 1877.

Beadlea longibracteata (Barb.Rodr.) Garay, Bot. Mus. Leafl. 28(4): 301.1982 (“1980”).

HABIT AND HABITAT: Rupicolous; riparian forest.

Voucher: Caetano 26 (FURB).

Cyclopogon multiflorus Schltr., Anexos Mem. Inst.

Butantan, Secç. Bot. 1(4): 27. 1922.*

Beadlea multiflora (Schltr.) Garay, Bot. Mus. Leafl. 28(4): 301. 1982 (“1980”).

HABIT AND HABITAT: Epiphyte; forest interior.

VOUCHER: Caetano \& Schlemper 198 (spirit, FURB).

Cyclopogon subalpestris Schltr., Repert. Spec. Nov. Regni Veg. Beih. 35: 32. 1925 (Fig. 5).

Beadlea subalpestris (Schltr.) Garay, Bot. Mus. Leafl. 28(4): 301. 1982 (“1980").

HABIT AND HABITAT: Rupicolous; forest interior (top of hill).

VOUCHER: Caetano \& Schlemper 25 (FURB).

Dichaea Lindl.

Dichaea cogniauxiana Schltr., Anexos Mem. Inst. Butantan, Secç. Bot. 1(4): 66. 1922 (Fig. 5).* HabiT AND HABITAT: Epiphyte; riparian forest. Vouchers: Caetano s.n. (FURB 40575); Caetano 60 (FURB).

Dichaea pendula (Aubl.) Cogn. in Urb., Symb. Antill. 
4(1): 182. 1903 (Fig. 5).

Limodorum pendulum Aubl., Hist. Pl. Guiane 2: 819. 1775.

Habit AND HABITAT: Epiphyte; riparian forest.

VOUCHER: Caetano 54 (FURB).

\section{Dryadella Luer}

Dryadella lilliputiana (Cogn.) Luer, Selbyana 2(2-3): 208. 1978 (Fig. 5). [VU]

Masdevallia lilliputiana Cogn. in Mart., Fl. Bras. 3(6): 555. 1906.

HABIT AND HABITAT: Epiphyte; forest interior.

VOUCHER: Schmitt et al. 1799 (FURB).

Dryadella zebrina (Porsch) Luer, Selbyana 2(2-3): 209. 1978.

Masdevallia zebrina Porsch, Oesterr. Bot. Z. 55: 154. 1905.

HABIT AND HABITAT: Epiphyte; riparian forest.

VOUCHER: Caetano s.n. (FURB 38755).

\section{Elleanthus C.Presl}

Elleanthus brasiliensis (Lindl.) Rchb.f. in Walp., Ann. Bot. Syst. 6(3): 475. 1862 (Fig. 5).

Evelyna brasiliensis Lind1., London J. Bot. 2: 661. 1843.

HABIT AND HABITAT: Rupicolous; forest edge.

VOUCHER: Caetano s.n. (FURB 36923).

\section{ENCYCLIA Hook.}

Encyclia patens Hook., Bot. Mag. 57: t. 3013. 1830 var. patens (Fig. 5).*

Epidendrum odoratissimum Lindl., Edwards's Bot. Reg. 17: t. 1415. 1831.

Encyclia odoratissima (Lindl.) Schltr., Orchideen: 210. 1914.

HaBiT AND HABITAT: Epiphyte; riparian forest.

VOUCHER: Caetano s.n. (FURB 20595).

\section{Epidendrum L.}

Epidendrum avicula Lindl., J. Bot. (Hooker) 3: 85. 1841. Lanium avicula (Lindl.) Benth., Hooker's Icon. P1. 14: t. 1335. 1881.

HABIT AND HABITAT: Epiphyte; riparian forest.

Voucher: Caetano 71 (FURB).

Epidendrum caldense Barb.Rodr., Gen. Spec. Orchid.

2: 148. 1882.*

HABIT AND HABITAT: Epiphyte; anthropic area (pasture).
Voucher: Caetano \& Almeida 201 (spirit, FURB).

Epidendrum campaccii Hágsater \& L.Sánchez, Icon.

Orchid. 2(1): t. 117. 1993.*

HABIT AND HABITAT: Epiphyte; open field.

Voucher: Caetano s.n. (FURB 37807).

Epidendrum cristatum Ruiz \& Pav., Syst. Veg. Fl.

Peruv. Chil. 1: 243. 1798.

Epidendrum raniferum Lindl., Gen. Sp. Orchid. P1.: 109. 1831.

HABIT AND HABITAT: Epiphyte; riparian forest.

VOUCHER: Caetano s.n. (FURB 37805).

Epidendrum densiflorum Hook., Bot. Mag. 66: t. 3791. 1840 (Fig. 6).

HABIT AND HABITAT: Epiphyte; forest interior.

VOUCHER: Caetano s.n. (FURB 38757).

Epidendrum latilabrum Lindl., Edwards's Bot. Reg. 27(Misc.): 77. 1841.

Amphiglottis latilabra (Lindl.) Acuña, Bol. Estac. Exp. Agron. Santiago de las Vegas 60: 98. 1939.

Neolehmannia latilabris (Lind1.) Pabst, Bradea 2(46): 306. 1978.

HABit AND HABITAT: Epiphyte; forest interior, riparian forest.

Vouchers: Caetano 18 (FURB); 189 (spirit, FURB).

Epidendrum proligerum Barb.Rodr., Gen. Spec.

Orchid. 1: 61. 1877.*

Epidendrum corymbosum Lindl., Fol. Orchid. 3(Epidendrum): 61. 1853, nom. illeg.

Epidendrum ochrochlorum Barb.Rodr., Gen. Spec.

Orchid. 2: 140. 1882.

HABIT AND HABITAT: Epiphyte; riparian forest.

Voucher: Caetano \& Schlemper 67 (FURB).

Epidendrum pseudodifforme Hoehne \& Schltr., Repert. Spec. Nov. Regni Veg. Beih. 35: 71. 1925.* HABIT AND HABITAT: Epiphyte; riparian forest.

VOUCHER: Crestani s.n. (FURB 8453).

Epidendrum ramosum Jacq., Enum. Syst. P1.: 29. 1760 (Fig. 6).

HABIT AND HABITAT: Epiphyte; riparian forest.

Vouchers: Caetano 32 (FURB); Caetano \& Schlemper s.n. (FURB 40578).

Epidendrum rigidum Jacq., Enum. Syst. P1.: 29. 1760.

Habit and habitat: Epiphyte; anthropic area (pasture).

Voucher: Caetano 187 (spirit, FURB).

Epidendrum secundum Jacq., Enum. Syst. P1.: 29. 1760

(Fig. 6). 
Epidendrum elongatum Jacq., Collecteana 3: 260. 1791.

Epidendrum ellipticum Graham in Hook., Exot. Fl. 3: t. 207. 1826.

Epidendrum ansiferum Rchb.f. \& Warsz., Bonplandia 2: 111. 1854.

HABIT AND HABITAT: Epiphyte; anthropic area (approach road).

Voucher: Caetano 17 (FURB).

Epidendrum tridactylum Lindl., Edwards's Bot. Reg. 24(Misc.): 46.1838 (Fig. 6).

Amblostoma tridactylum (Lindl.) Rchb.f. in Walp., Ann. Bot. Syst. 6(4): 485. 1863.

HABIT AND HABITAT: Epiphyte; forest interior.

VOUCHer: Caetano 20 (FURB).

Epidendrum vesicatum Lindl., Edwards's Bot. Reg. 24(Misc.): 50.1838 (Fig. 6).*

HABIT AND HABITAT: Epiphyte; riparian forest.

Voucher: Caetano \& Schlemper 68 (FURB).

Eulophia R.Br.

Eulophia alta (L.) Fawc. \& Rendle, Fl. Jamaica 1: 112. 1910 (Fig. 6).

Limodorum altum L., Syst. Nat. ed. 12. 2: 594. 1767. Eulophia longifolia (Kunth) Schltr., Orchideen: 347. 1914.

Dendrobium longifolium Kunth, Nov. Gen. Sp. 1(ed. qu.): 360. 1816.

HABIT AND HABITAT: Terrestrial; anthropic area (pasture).

Voucher: Caetano 61 (FURB).

\section{Eurystyles Wawra}

Eurystyles cotyledon Wawra, Oesterr. Bot. Z. 13(1): 223. 1863 (Fig. 6).

HABIT AND HABITAT: Epiphyte; riparian forest (forest edge), anthropic area (pasture).

Vouchers: Caetano s.n. (FURB 40579); Caetano 220 (spirit, FURB).

\section{GoMesA R.Br.}

Gomesa albinoi (Schltr.) M.W.Chase \& N.H.Williams, Ann. Bot. (Oxford) 104(3): 395. 2009.*

Oncidium albinoi Schltr., Repert. Spec. Nov. Regni Veg. 23: 63. 1926.

Baptistonia albinoi (Schltr.) Chiron \& V.P.Castro, Richardiana 4(3): 117. 2004.
HABit AND HABITAT: Epiphyte; riparian forest, anthropic area (pasture).

Vouchers: Caetano \& Schlemper 203 (spirit, FURB); Caetano \& Schlemper 206 (spirit, FURB).

Gomesa cornigera (Lindl.) M.W.Chase \& N.H. Williams, Ann. Bot. (Oxford) 104(3): 396. 2009.

Oncidium cornigerum Lindl., Edwards's Bot. Reg. 18: t. 1542. 1832.

Baptistonia cornigera (Lindl.) Chiron \& V.P.Castro, Richardiana 4(3): 117. 2004.

Oncidium hecatanthum Kraenzl., Kungl. Svenska Vetenskapsakad. Handl., n.s. 46(10): 81. 1911.

HABIT AND HABITAT: Epiphyte; anthropic area (urban area).

VOUCHER: Caetano s.n. (FURB 37361).

Gomesa eleutherosepala (Barb.Rodr.) M.W.Chase \& N.H.Williams, Ann. Bot. (Oxford) 104(3): 396. 2009.*

Rodriguezia eleutherosepala Barb.Rodr., Gen. Spec. Orchid. 2: 240. 1882.

Rodrigueziopsis eleutherosepala (Barb.Rodr.) Schltr., Repert. Spec. Nov. Regni Veg. 16: 427. 1920.

HABIT AND HABITAT: Epiphyte; riparian forest, forest interior.

Vouchers: Schmitt et al. 1740 (FURB); Caetano s.n. (FURB 37333).

Gomesaflexuosa (Lodd.) M.W.Chase \& N.H.Williams, Phytotaxa 1: 58. 2009 (Fig. 6).

Oncidium flexuosum Lodd., Bot. Cab. 5: t. 424. 1820.

Ampliglossum flexuosum (Lodd.) Campacci, Colet. Orquídeas Brasil. 3: 84. 2006.

Coppensia flexuosa (Lodd.) Campacci, Bol. CAOB 62: 55. 2006.

HABIT AND HABITAT: Epiphyte; forest interior (riparian forest).

VOUCHER: Caetano s.n. (FURB 36893).

Gomesa gardneri (Lindl.) M.W.Chase \& N.H.Williams, Ann. Bot. (Oxford) 104(3): 397. 2009 (Fig. 6).*

Oncidium gardneri Lindl., London J. Bot. 2: 662. 1843.

Anettea gardneri (Lindl.) Szlach. \& Mytnik, Polish Bot. J. 51(1): 50. 2006.

Brasilidium gardneri (Lindl.) Campacci, Colet. Orquídeas Brasil. 3: 79. 2006. 

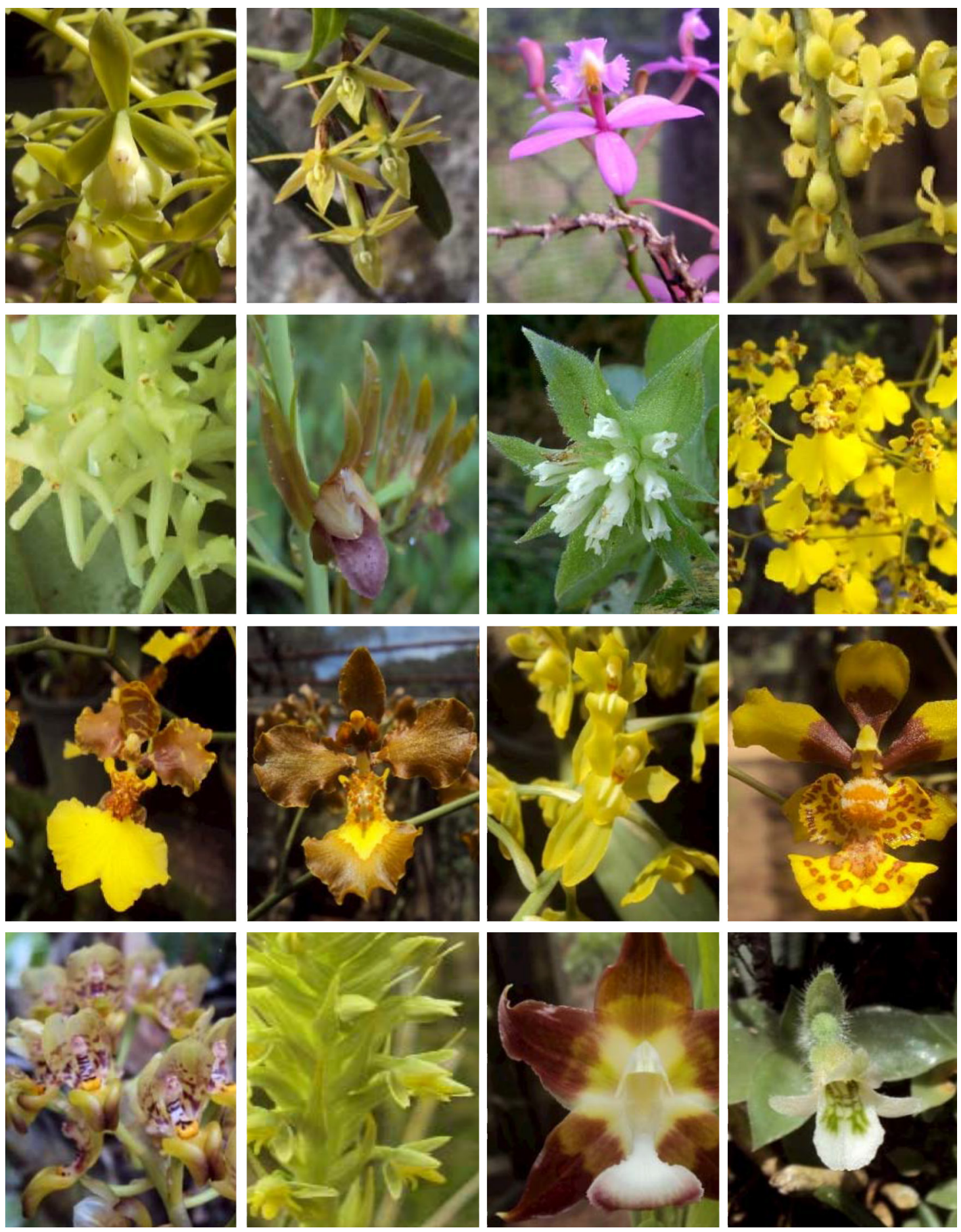

FiguRE 6. From top left to bottom right: Epidendrum densiflorum. E. ramosum. E. secundum. E. tridactylum. E. vesicatum. Eulophia alta. Eurystyles cotyledon. Gomesa flexuosa. G. gardneri. G. imperatoris-maximiliani. G. recurva. Grandiphyllum divaricatum. Grobya fascifera. Habenaria parviflora. Huntleya meleagris. Lankesterella ceracifolia. All courtesy of The Field Museum, Chicago. 

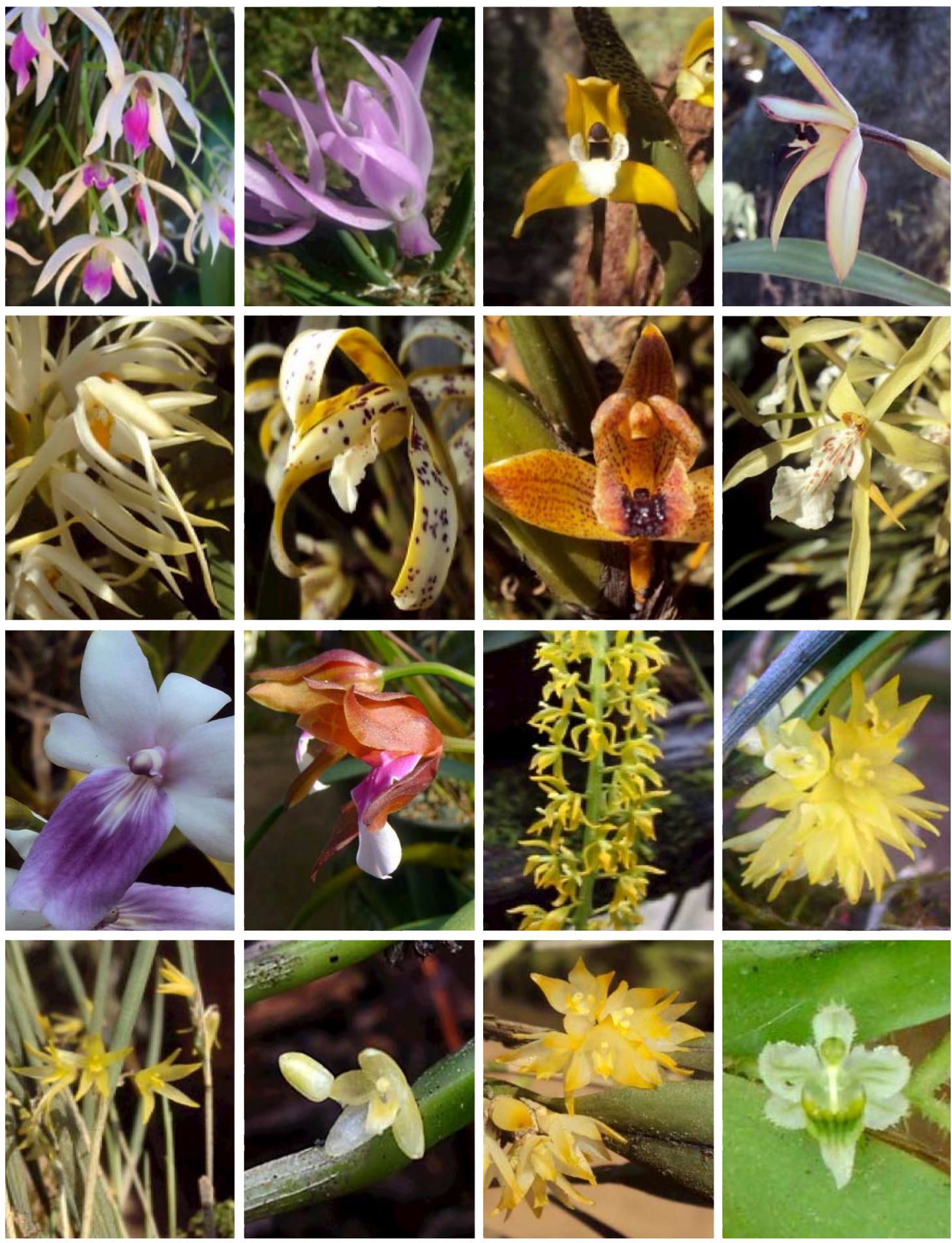

FIgURE 7. From top left to bottom right: Leptotes bicolor. L. unicolor. Maxillaria chrysantha. M. marginata. M. ochroleuca. M. picta. M. subulata. Miltonia falvescens. M. regnellii. M. russelliana. Notylia hemitricha. Octomeria crassifolia, O. gracilis. O. micrantha. O. warmingii. Ornithocephalus myrticola. All courtesy of The Field Museum, Chicago. 
HABIT AND HABITAT: Epiphyte; riparian forest, anthropic area (pasture).

Vouchers: Schmitt et al. 506 (FURB); Schmitt et al. 515 (FURB); Caetano \& Schlemper 202 (spirit, FURB).

Gomesa handroi (Hoehne) Pabst, Orquídea (Rio de Janeiro) 29(4): 165. 1967.*

Theodorea handroi Hoehne, Arq. Bot. Estado São Paulo, n.s. 1(4): 87. 1942.

Hellerorchis handroi (Hoehne) A.D.Hawkes, Orchid J. 3: 275. 1959.

Rodrigueziella handroi (Hoehne) Pabst, Bradea 2(2): 8. 1975.

HABIT AND HABITAT: Epiphyte; riparian forest.

VOUCHER: Caetano s.n. (FURB 36288).

Gomesa imperatoris-maximiliani (Rchb.f.) M.W.Chase \& N.H.Williams, Ann. Bot. (Oxford) 104(3): 397. 2009 (Fig. 6).*

Oncidium imperatoris-maximiliani Rchb.f. in Wawra, Bot. Ergebn.: 154. 1866.

Anettea imperatoris-maximiliani (Rchb.f.) Szlach. \& Mytnik, Polish Bot. J. 51(1): 50. 2006.

Oncidium crispum Lodd. ex Lindl., Gen. Sp. Orchid. P1.: 197. 1833.

Anettea crispa (Lodd. ex Lindl.) Szlach. \& Mytnik, Polish Bot. J. 5(1)1: 50. 2006.

Brasilidium crispum (Lodd. ex Lindl.) Campacci, Colet. Orquídeas Brasil. 3: 78. 2006.

Oncidium brunnipetalum Barb.Rodr., Gen. Spec. Orchid. 2: 190. 1882.

Ampliglossum brunnipetalum (Barb.Rodr.) Campacci, Colet. Orquídeas Brasil. 3: 83. 2006.

Coppensia brunnipetala (Barb.Rodr.) Campacci, Bol. CAOB 62: 55. 2006.

Gomesa brunnipetala (Barb.Rodr.) M.W.Chase \& N.H.Williams, Ann. Bot. (Oxford) 104(3): 396. 2009. HABIT AND HABITAT: Epiphyte; anthropic area (pasture). Voucher: Caetano s.n. (FURB 37814).

Gomesa loefgrenii (Cogn.) M.W.Chase \& N.H.Williams, Ann. Bot. (Oxford) 104(3): 397. 2009.*

Oncidium loefgrenii Cogn. in Mart., F1. Bras. 3(4): 381. 1896.

Carenidium loefgrenii (Cogn.) Baptista, Colet. Orquídeas Brasil. 3: 91. 2006.

Menezesiella loefgrenii (Cogn.) V.P.Castro \& Chiron, Richardiana 6(2): 105. 2006.
Coppensia loefgrenii (Cogn.) F.Barros \& V.T.Rodrigues, Bol. CAOB 77-78: 12. 2010.

HABIT AND HABITAT: Epiphyte; riparian forest.

VOUCHER: Klein \& Bresolin 10882 (FLOR).

Gomesa radicans (Rchb.f.) M.W.Chase \& N.H.Williams, Ann. Bot. (Oxford) 104(3): 398. 2009.*

Sigmatostalix radicans Rchb.f. in Walp., Ann. Bot. Syst. 6(6): 859. 1864.

Ornithophora radicans (Rchb.f.) Garay \& Pabst, Orquídea (Rio de Janeiro) 13: 50. 1951.

HABIT AND HABITAT: Epiphyte; riparian forest.

VOUCHER: Caetano s.n. (FURB 37804).

Gomesa ranifera (Lindl.) M.W.Chase \& N.H.Williams, Ann. Bot. (Oxford) 104(3): 398. 2009.*

Oncidium raniferum Lindl., Edwards's Bot. Reg. 23: t. 1920. 1837.

Carenidium raniferum (Lindl.) Baptista, Colet. Orquídeas Brasil. 3: 91. 2006.

Menezesiella ranifera (Lindl.) Chiron \& V.P.Castro, Richardiana 6(2): 105. 2006.

Rhinocidium raniferum (Lindl.) Baptista, Orchidstud. 2(2-3): 42. 2007.

Coppensia ranifera (Lindl.) F.Barros \& V.T.Rodrigues, Bol. CAOB 77-78: 13. 2010.

HABIT AND HABITAT: Epiphyte; forest interior, anthropic area (pasture).

Vouchers: Schmitt et al. 1798 (FURB); Caetano \& Schlemper 205 (spirit, FURB).

Gomesa recurva R.Br., Bot. Mag. 42: t. 1748. 1815. Rodriguezia barkeri Hook., Bot. Mag. 54: t. 2746. 1827 (Fig. 6).

Odontoglossum barkeri (Hook.) Rchb.f. in Walp., Ann. Bot. Syst. 6(6): 854. 1864.

Gomesa barkeri (Hook.) Rolfe, Orchid Rev. 9: 166. 1901.

HABIT AND HABITAT: Epiphyte; riparian forest, forest interior.

Vouchers: Bresolin 665 (FLOR, as Gomesa barkeri); Caetano s.n. (FURB 37353).

Gomesa riograndensis (Cogn.) M.W.Chase \& N.H.Williams, Ann. Bot. (Oxford) 104(3): 398. 2009.

Oncidium riograndense Cogn. in Mart., F1. Bras. 3(6): 446. 1906.

Baptistonia riograndense (Cogn.) Chiron \& V.P.Castro, Richardiana 4(3): 118. 2004. 
HABIT AND HABITAT: Epiphyte; riparian forest.

VOUCHER: Crestani s.n. (FURB 8454).

Gomesa uniflora (Booth ex Lindl.) M.W.Chase \& N.H.Williams, Ann. Bot. (Oxford) 104(3): 398. 2009. Oncidium uniflorum Booth ex Lindl., Edwards's Bot. Reg. 29: t. 43. 1843.

Alatiglossum uniflorum (Booth ex Lindl.) Baptista, Colet. Orquídeas Brasil. 3: 89. 2006.

Kleberiella uniflora (Booth ex Lind1.) V.P.Castro \& Cath., Richardiana 6(3): 158. 2006.

Oncidium longipes Lindl., Paxton's Fl. Gard. 1: 46. 1850 .

Alatiglossum longipes (Lindl.) Baptista, Colet. Orquídeas Brasil. 3: 88. 2006.

Kleberiella longipes (Lindl.) V.P.Castro \& Cath., Richardiana 6(3): 159. 2006.

Gomesa longipes (Lindl.) M.W.Chase \& N.H.Williams, Ann. Bot. (Oxford) 104(3): 397. 2009.

HABIT AND HABITAT: Epiphyte; forest edge.

Voucher: Caetano s.n. (FURB 37350).

\section{Gongora Ruiz \& Pav.}

Gongora bufonia Lindl., Edwards's Bot. Reg. 27: t. 2. 1841.*

HABIT AND HABITAT: Epiphyte; forest interior (top of hill).

Voucher: Caetano \& Henschel 53 (FURB).

\section{GovenIa Lindl.}

Govenia utriculata (Sw.) Lindl., Edwards's Bot. Reg. 25(Misc.): 47. 1839.

Limodorum utriculatum Sw., Prodr.: 119. 1788.

HABIT AND HABITAT: Terrestrial; forest edge.

VOUCher: Caetano 75 (FURB, RB).

\section{GrandiPhyllum Docha Neto}

Grandiphyllum divaricatum (Lind1.) Docha Neto, Colet. Orquídeas Brasil. 3: 75. 2006 (Fig. 6). [VU] Oncidium divaricatum Lindl., Bot. Reg. 13: t. 1050. 1827.

Aurinocidium divaricatum (Lindl.) Romowicz \& Szlach., Polish Bot. J. 51(1): 44. 2006.

Oncidium pulvinatum Lindl., Edwards's Bot. Reg. 24(Misc.): 61. 1838.

Aurinocidium pulvinatum (Lindl.) Romowicz \& Szlach., Polish Bot. J. 51(1): 44. 2006.
Grandiphyllum pulvinatum (Lind1.) Docha Neto, Colet. Orquídeas Brasil. 3: 76. 2006.

Oncidium sphegiferum Lindl., Edwards's Bot. Reg. 29(Misc.): 17. 1843.

Aurinocidium sphegiferum (Lindl.) Romowicz \& Szlach., Polish Bot. J. 51(1): 44. 2006.

Grandiphyllum sphegiferum (Lindl.) Docha Neto, Colet. Orquídeas Brasil. 3: 76. 2006.

Oncidium robustissimum Rchb.f., Gard. Chron., ser. 3, 4: 352. 1888.

Aurinocidium robustissimum (Rchb.f.) Romowicz \& Szlach., Polish Bot. J. 51(1): 44. 2006.

Grandiphyllum robustissimum (Rchb.f.) Docha Neto, Colet. Orquídeas Brasil. 3: 76. 2006.

HABIT AND HABITAT: Epiphyte; anthropic area (urban area).

VOUCHer: Caetano s.n. (FURB 39665).

Grandiphyllum hians (Lindl.) Docha Neto, Colet. Orquídeas Brasil. 3: 75. 2006.* [VU]

Oncidium hians Lindl., Edwards's Bot. Reg. 24(Misc.): 65.1838.

Aurinocidium hians (Lindl.) Romowicz \& Szlach., Polish Bot. J. 51(1): 44. 2006.

HABIT AND HABITAT: Epiphyte; riparian forest.

VOUCHER: Caetano 57 (FURB).

\section{Grobya Lindl.}

Grobya fascifera Rchb.f., Flora 69: 551. 1886. [VU] Grobya bibrachiata Hoehne, Bol. Agric. (São Paulo) 34: 625. 1934 (Fig. 6).*

Grobya bibrachiata var. riograndensis Pabst, Arch. Jard. Bot. Rio de Janeiro 14: 22. 1956.

HABIT AND HABITAT: Epiphyte; forest interior.

Voucher: Caetano 70 (FURB).

\section{Habenaria Willd.}

Habenaria josephensis Barb.Rodr., Gen. Spec. Orchid. 2: 257. 1882.*

HABIT AND HABITAT: Terrestrial; forest interior.

Voucher: Caetano 9 (FURB).

Habenaria parviflora Lindl., Gen. Sp. Orchid. P1.: 314. 1835 (Fig. 6).

Habit and habitat: Terrestrial; anthropic area (pasture).

Vouchers: Costa s.n. (R 90253); Caetano 55 (FURB). 


\section{HaPalORChis Schltr.}

Hapalorchis lineata (Lindl.) Schltr., Beih. Bot. Centralbl. 37(2): 363. 1920.

Spiranthes lineata Lindl., Gen. Sp. Orchid. P1.: 471. 1840.

Gyrostachys lineata (Lindl.) Kuntze, Revis. Gen. P1. 2: 664. 1891.

Cyclopogon lineatus (Lindl.) Pabst, Bradea 1(47): 466. 1974.

HABIT AND HABITAT: Terrestrial; forest interior.

VOUCHER: Caetano 212 (spirit, FURB).

Hapalorchis micranthus (Barb.Rodr.) Hoehne, Fl. Brasilica 12(2): 291.1945 (“micrantha”).

Spiranthes micrantha Barb.Rodr., Gen. Spec. Orchid. 1: 183. 1877.

Cyclopogon micranthus Barb.Rodr., Gen. Spec. Orchid. 1(Index): iii. 1877.

Sauroglossum candidum Kraenzl., Kungl. Svenska Vetenskapsakad. Handl., n.s. 46(10): 38. 1911.

Hapalorchis candida (Kraenzl.) Schltr., Beih. Bot. Centralbl. 37(2): 363. 1920.

Cyclopogon candidus (Kraenzl.) Pabst, Bradea 1(47): 466. 1974.

HABIT AND HABITAT: Terrestrial; riparian forest.

VOUCHER: Schmitt et al. 504 (FURB).

Huntleya Bateman ex Lindl.

Huntleya meleagris Lindl., Edwards's Bot. Reg. 23: t. 1991.1837.

HABIT AND HABITAT: Epiphyte; riparian forest.

Vouchers: Caetano 65 (FUEL, FURB).

IsABELIA Barb.Rodr.

Isabelia pulchella (Kraenzl.) Van den Berg \& M.W.Chase, Lindleyana 16(2): 109. 2001.*

Neolauchea pulchella Kraenzl., Bull. Herb. Boissier 5: 110. 1897.

HABIT AND HABITAT: Epiphyte; riparian forest.

VOUChER: Caetano 16 (FURB).

\section{IsochILus R.Br.}

Isochilus linearis (Jacq.) R.Br. in W.T.Aiton, Hortus Kew., ed. 2. 5: 209. 1813.

Epidendrum lineare Jacq., Enum. Syst. Pl.: 29. 1760.

Isochilus brasiliensis Schltr., Arch. Bot. São Paulo 1: 235.1926.
HABIT AND HABITAT: Epiphyte; anthropic area (urban area).

VOUCHER: Caetano s.n. (FURB 38753).

\section{LanKesterella Ames}

Lankesterella ceracifolia (Barb.Rodr.) Mansf., Notizbl. Bot. Gart. Berlin-Dahlem 15: 217. 1940. Stenorrhynchos ceracifolium Barb.Rodr., Gen. Spec. Orchid. 2(Index): xv. 1881.

Spiranthes ceracifolia (Barb.Rodr.) Barb.Rodr., Gen. Spec. Orchid. 2: 285. 1882.

HABIT AND HABITAT: Epiphyte; forest edge, forest interior.

Vouchers: Schmitt et al. 1810 (FURB); Caetano 73 (FURB).

\section{LEPTOTES Lindl.}

Leptotes bicolor Lindl., Edwards's Bot. Reg. 19: t. 1625. 1833 (Fig. 7).

HABIT AND HABITAT: Epiphyte; riparian forest.

Voucher: Caetano 37 (FURB).

Leptotes unicolor Barb.Rodr., Gen. Spec. Orchid. 1: 74. 1877 (Fig. 7).

HABIT AND HABITAT: Epiphyte; anthropic area (pasture).

VOUCher: Caetano s.n. (FURB 38094).

LIPARIS Rich.

Liparis nervosa (Thunb.) Lindl., Gen. Sp. Orchid. P1.: 26. 1830 subsp. nervosa.

Ophrys nervosa Thunb. in Murray, Syst. Veg. ed. 14: 814. 1784.

Liparis elata Lindl., Bot. Reg. 14: t. 1175. 1828.

HABIT AND HABITAT: Terrestrial; forest interior.

Voucher: Caetano 3 (FURB).

Malaxis Sol. ex Sw.

Malaxis excavata (Lindl.) Kuntze, Revis. Gen. Pl. 2: 673. 1891

Microstylis excavata Lindl., Edwards's Bot. Reg. 24(Misc.): 51. 1838.

Cheiropterocephalus sertuliferus Barb.Rodr., Gen. Spec. Orchid. 1: 29. 1877.

Microstylis sertulifera (Barb.Rodr.) Schltr., Repert. Spec. Nov. Regni Veg. Beih. 35: 46. 1925.

Malaxis sertulifera (Barb.Rodr.) Pabst, Orquídea (Rio de Janeiro) 29(3): 112. 1967. 
HABIT AND HABITAT: Epiphyte; forest edge.

Voucher: Caetano 35 (FURB).

Malaxis parthonii C.Morren, Bull. Acad. Roy. Sci. Bruxelles 5: 485. 1838.

Microstylis parthonii (C.Morren) Rchb.f., Ann. Bot. Syst. 6: 206. 1861

HABIT AND HABITAT: Terrestrial; riparian forest.

Voucher: Caetano s.n. (FURB 40572).

Maxillaria Ruiz \& Pav.

Maxillaria brasiliensis Brieger \& Illg, Trab. 26 Congr. Nac. Bot., Rio de Janeiro: 240. 1977.*

Heterotaxis brasiliensis (Brieger \& Illg) F.Barros, Hoehnea 29(2): 112. 2002.

HABIT AND HABITAT: Epiphyte; riparian forest.

VOUCHER: Caetano 181 (spirit, FURB).

Maxillaria chrysantha Barb.Rodr., Gen. Spec. Orchid. 1: 115. 1877 (Fig. 7).

Brasiliorchis chrysantha (Barb.Rodr.) R.B.Singer, S.Koehler \& Carnevali, Novon 17(1): 96. 2007.

Bolbidium chrysanthum (Barb.Rodr.) J.M.H.Shaw, Orchid Rev. Suppl. 119(1294): 38. 2011.

Maxillaria serotina Regnell \& Barb.Rodr., Gen. Spec. Orchid. 2: 203. 1882.

Brasiliorchis serotina (Regnell \& Barb.Rodr.) Szlach. \& Sitko, Biodivers. Res. Conservation 25: 23. 2012.

Habit AND HABITAT: Epiphyte; anthropic area (pasture).

VOUCHER: Caetano 42 (FURB).

Maxillaria cleistogama Brieger \& Illg, Trab. 26 Congr. Nac. Bot., Rio de Janeiro: 247. 1977.

Mormolyca cleistogama (Brieger \& Illg) M.A.Blanco, Lankesteriana 7(3): 531. 2007.

HABIT AND HABITAT: Epiphyte; riparian forest.

Voucher: Caetano s.n. (FURB 36888).

Maxillaria crocea Lindl., Edwards's Bot. Reg. 21: t. 1799. 1835.*

Maxillaria lindleyana Schltr., Repert. Spec. Nov. Regni Veg. Beih. 9: 162. 1921, nom. illeg. HaBiT AND HABITAT: Epiphyte; forest interior. Voucher: Caetano et al. 222 (spirit, FURB).

Maxillaria ferdinandiana Barb.Rodr., Gen. Spec. Orchid. 2: 204. 1882.*

Christensonella ferdinandiana (Barb.Rodr.)

Szlach. et al., Polish Bot. J. 51(1): 58. 2006.

HABIT AND HABITAT: Epiphyte; riparian forest.

VOUCHER: Caetano 23 (FURB).
Maxillaria marginata (Lind1.) Fenzl, Fl. Serres Jard. Eur. 10: 112. 1855 (Fig. 7).*

Cymbidium marginatum Lindl., Edwards's Bot. Reg. 18: t. 1530. 1832.

Brasiliorchis marginata (Lindl.) R.B.Singer, S.Koehler \& Carnevali, Novon 17(1): 97. 2007.

Bolbidium marginatum (Barb.Rodr.) J.M.H.Shaw, Orchid Rev. Suppl. 119(1294): 38. 2011.

Maxillaria consanguinea Klotzsch, Ann. Sci. Nat., Bot., sér. 3, 19: 375. 1853.

Brasiliorchis consanguinea (Klotzsch) R.B.Singer, S.Koehler \& Carnevali, Novon 17(1): 96. 2007.

HABIT AND HABITAT: Epiphyte; riparian forest.

Vouchers: Caetano s.n. (FURB 36932); Caetano s.n. (FURB 37343).

Maxillaria notylioglossa Rchb.f., Bonplandia 2(2): 16. 1854.

Rhetinantha notylioglossa (Rchb.f.) M.A.Blanco, Lankesteriana 7(3): 535. 2007.

Maxillaria cerifera Barb.Rodr., Gen. Spec. Orchid. 1: 118. 1877.

Ornithidium ceriferum (Barb.Rodr.) Barb.Rodr., Gen. Spec. Orchid. 2: 209. 1882.

Rhetinantha cerifera (Barb.Rodr.) M.A.Blanco, Lankesteriana 7(3): 534. 2007.

HABIT AND HABITAT: Epiphyte; riparian forest.

VOUCHER: Caetano s.n. (FURB 39736).

Maxillaria ochroleuca G.Lodd. ex Lindl., Gen. Sp. Orchid. Pl.: 143. 1832 (Fig. 7).

HABIT AND HABITAT: Epiphyte; riparian forest.

Voucher: Caetano s.n. (FURB 37811).

Maxillaria paranaensis Barb.Rodr., Gen. Spec. Orchid. 2: 205. 1882.

Christensonella paranaensis (Barb.Rodr.) Szlach. \& Sitko, Biodivers. Res. Conservation 25: 27. 2012.

Maxillaria spegazziniana Kraenzl., Orchis 2: 51. 1908.

Christensonella spegazziniana (Kraenzl.) Szlach. \& Sitko, Biodivers. Res. Conservation 25: 27. 2012.

Maxillaria juergensii Schltr., Repert. Spec. Nov. Regni Veg. Beih. 35: 88. 1925.

Christensonella juergensii (Schltr.) Szlach. et al., Polish Bot. J. 51(1): 58. 2006.

Maxillaria cogniauxiana Hoehne, Bol. Agric. (São Paulo) 34: 632. 1934 
Christensonella cogniauxiana (Hoehne) Szlach. et al., Polish Bot. J. 51(1): 58. 2006.

Maxillaria heterophylla Hoehne, Arq. Bot. Estado São Paulo, n.s. 2(6): 132. 1952.

Habit AND HABITAT: Epiphyte; forest edge.

VOUCHER: Caetano s.n. (FURB 37800).

Maxillaria picta Hook., Bot. Mag. 59: t. 3154.1832 (Fig. 7).

Brasiliorchis picta (Hook.) R.B.Singer, S.Koehler \& Carnevali, Novon 17(1): 97. 2007.

Bolbidium pictum (Barb.Rodr.) J.M.H.Shaw, Orchid Rev. Suppl. 119(1294): 38. 2011.

HABIT AND HABITAT: Epiphyte; riparian forest.

VOUCHER: Caetano s.n. (FURB 38760).

Maxillaria porphyrostele Rchb.f., Gard. Chron. 1: 978. 1873.*

Brasiliorchis porphyrostele (Rchb.f.) R.B.Singer, S.Koehler \& Carnevali, Novon 17(1): 97. 2007.

Bolbidium porphyrostele (Rchb.f.) J.M.H.Shaw, Orchid Rev. Suppl. 119(1294): 38. 2011.

HABIT AND HABITAT: Epiphyte; anthropic area (pasture).

VOUCHER: Caetano 207 (spirit, FURB).

Maxillaria rufescens Lindl., Edwards's Bot. Reg. 22: t. 1848.1836.

Mormolyca rufescens (Lindl.) M.A.Blanco, Lankesteriana 7(3): 531. 2007.

HABIT AND HABITAT: Epiphyte; riparian forest.

VOUCHER: Caetano 72 (FURB).

Maxillaria subulata Lindl., Gen. Sp. Orchid. Pl.: 147. 1832 (Fig. 7).*

Christensonella subulata (Lindl.) Szlach. et al., Polish Bot. J. 51(1): 59. 2006.

Maxillaria acicularis Herb. ex Lindl., Edwards's Bot. Reg. 23: t. 1986. 1837.

Christensonella acicularis (Herb. ex Lindl.) Szlach. et al., Polish Bot. J. 51(1): 58. 2006.

Maxillaria madida Lindl., Edwards's Bot. Reg. 24(Misc.): 44. 1838.

Christensonella madida (Lindl.) Szlach. et al., Polish Bot. J. 51(1): 58. 2006.

Maxillaria cepula Rchb.f., Bonplandia 3(15-16): 216. 1855.

Christensonella cepula (Rchb.f.) S.Koehler, Lankesteriana 7(3): 522. 2007.

Maxillaria mosenii Kraenzl., Kungl. Svenska Vetenskapsakad. Handl. 46(10): 73. 1911.
Maxillaria echinochila Kraenzl., Ark. Bot. 16(8): 22. 1921.

Maxillaria paulistana Hoehne, Arq. Bot. Estado São Paulo, n.s. 2(6): 135. 1952.

Christensonella paulistana (Hoehne) Szlach. et al., Polish Bot. J. 51(1): 58. 2006.

HABIT AND HABITAT: Epiphyte; riparian forest.

Voucher: Caetano 43 (FURB).

\section{Microchilus C.Presl}

Microchilus arietinus (Rchb.f. \& Warm.) Ormerod, Lindleyana 17(4): 214. 2002.

Physurus arietinus Rchb.f. \& Warm. in Rchb.f., Otia Bot. Hamburg. 2: 82. 1881.

Erythrodes arietina (Rchb.f. \& Warm.) Ames, Orchidaceae 7: 66. 1922.

HABIT AND HABITAT: Terrestrial; riparian forest.

VOUCHER: Caetano 183 (spirit, FURB).

Miltonia Lindl.

Miltonia flavescens (Lindl.) Lindl., Sert. Orchid.: t. 48. 1841 (Fig. 7).

Cyrtochilum flavescens Lindl., Edwards's Bot. Reg. 19: t. 1627. 1834.

HABIT AND HABITAT: Epiphyte; anthropic area (urban area).

Voucher: Caetano 44 (FURB).

Miltonia regnellii Rchb.f., Linnaea 22: 851.1850 (Fig. 7).*

HaBIT AND HABITAT: Epiphyte; riparian forest.

Voucher: Caetano s.n. (FURB 37808).

Miltonia russelliana (Lindl.) Lindl., Sert. Orchid.: t. 48. 1841 (Fig. 7).*

Oncidium russellianum Lindl., Edwards's Bot. Reg. 22: t. 1830. 1836.

HABIT AND HABITAT: Epiphyte; riparian forest.

VOUCHER: Caetano 19 (FURB).

Myoxanthus Poepp. \& Endl.

Myoxanthus exasperatus (Lindl.) Luer, Selbyana 7(1): 36. 1982.

Pleurothallis exasperata Lindl., Fol. Orchid. 9(Pleurothallis): 15. 1859.

Humboltia exasperata (Lindl.) Kuntze, Revis. Gen. Pl. 2: 667. 1891.

Pleurothallis peduncularis Lindl., Edwards's Bot. Reg. 29(Misc.): 47. 1843, nom. illeg. 
Humboltia peduncularis (Lindl.) Kuntze, Revis. Gen. P1. 2: 668. 1891, nom. illeg.

Specklinia peduncularis (Lind1.) F.Barros, Hoehnea 10: 110. 1984, nom. illeg.

HABIT AND HABITAT: Epiphyte; riparian forest.

Voucher: Caetano 193 (spirit, FURB).

\section{Notylia Lindl.}

Notylia hemitricha Barb.Rodr., Gen. Spec. Orchid. 2: 223. 1882 (Fig. 7).*

HABIT AND HABITAT: Epiphyte; riparian forest.

VOUCher: Caetano s.n. (FURB 37802).

\section{Octomeria R.Br.}

Octomeria crassifolia Lindl., Companion Bot. Mag. 2: 354. 1836 (Fig. 7).

Octomeria alpina Barb.Rodr., Gen. Spec. Orchid. 2: 102.1881.

Octomeria densiflora Barb.Rodr., Gen. Spec. Orchid. 2: 97. 1881.

Octomeria gracilicaulis Schltr., Repert. Spec. Nov. Regni Veg. Beih. 35: 63. 1925.

HABIT AND HABITAT: Epiphyte; riparian forest.

Vouchers: Caetano 28(FURB); Caetano 30 (FURB).

Octomeria diaphana Lindl., Edwards's Bot. Reg. 25(Misc.): 91. 1839.*

Octomeria fialhoensis Dutra ex Pabst, Sellowia 10: 133. 1959.

HABIT AND HABITAT: Epiphyte; riparian forest.

VOUCHer: Caetano s.n. (FURB 38350).

Octomeria gracilis Lodd. ex Lindl., Edwards's Bot. Reg. 24(Misc.): 36.1838 (Fig. 7).*

HABIT AND HABITAT: Epiphyte; riparian forest.

Voucher: Caetano s.n. (FURB 36292).

Octomeria grandiflora Lindl., Edwards's Bot. Reg. 28(Misc.): 64. 1842.

Octomeria robusta Barb.Rodr., Gen. Spec. Orchid.

2: 97. 1881, nom. illeg.

Octomeria grandiflora var. robusta (Barb.Rodr.)

Cogn. in Mart., Fl. Bras. 3(4): 606. 1896.

HABIT AND HABITAT: Epiphyte; riparian forest.

Voucher: Caetano s.n. (FURB 36925).

Octomeria micrantha Barb.Rodr., Gen. Spec. Orchid. 1: 33.1877 (Fig. 7).

HABIT AND HABITAT: Epiphyte; anthropic area (reforested).

VOUCHER: Caetano 29 (FURB).
Octomeria octomeriantha (Hoehne) Pabst, Bradea 1(20): 180. 1972.*

Pleurothallis octomeriantha Hoehne, Bol. Mus. Nac. Rio de Janeiro 12(2): 24. 1936.

HaBit AND HABITAT: Epiphyte; anthropic area (reforested).

Voucher: Caetano 210 (spirit, FURB).

Octomeria palmyrabellae Barb.Rodr., Rodriguésia 8: 38. 1937.*

HaBit AND HABITAT: Epiphyte; anthropic area (reforested).

Voucher: Caetano \& Henschel 74 (FURB).

Octomeria pusilla Lindl., Companion Bot. Mag. 2: 354. 1837.*

Octomeria umbonulata Schltr., Repert. Spec. Nov. Regni Veg. Beih. 35: 67. 1925.

HABIT AND HABITAT: Epiphyte; anthropic area (pasture).

VOUCHER: Caetano \& Schlemper 196 (spirit, FURB).

Octomeria warmingii Rchb.f., Otia Bot. Hamburg.: 94. 1881 (Fig. 7).

Octomeria oxychela Barb.Rodr., Gen. Spec. Orchid. 2: 99. 1881.

HABIT AND HABITAT: Epiphyte; riparian forest.

VOUCHER: Caetano 52 (FURB).

\section{OrNithocephaLus Hook.}

Ornithocephalus myrticola Lindl., Ann. Nat. Hist. 4(26): 383.1840 (Fig. 7).

HABIT AND HABITAT: Epiphyte; riparian forest.

Vouchers: Caetano s.n. (FURB 37875); Royer 114 (UPCB).

\section{Pabstiella Brieger \& Senghas}

Pabstiella bicolor (Barb.Rodr.) Luer \& Toscano, Harvard Pap. Bot. 16(2): 380. 2011. [Luer \& Toscano de Brito 2011].*

Lepanthes bicolor Barb.Rodr., Gen. Spec. Orchid. 2: 49. 1881.

Pleurothallis bicolor (Barb.Rodr.) Cogn. in Mart., Fl. Bras. 3(4): 408. 1896, nom. illeg.

Trichosalpinx bicolor (Barb.Rodr.) Luer, Phytologia 54(5): 394. 1983.

Lepanthes quadridentata Barb.Rodr., Gen. Spec. Orchid. 2: 50. 1881.

Pleurothallis quadridentata (Barb.Rodr.) Cogn. in Mart., Fl. Bras. 3(4): 454. 1896. 
Trichosalpinx quadridentata (Barb.Rodr.) Luer, Phytologia 54(5): 397. 1983.

Specklinia quadridentata (Barb.Rodr.) Luer, Monogr. Syst. Bot. Missouri Bot. Gard. 95: 263. 2004. Pabstiella quadridentata (Barb.Rodr.) Luer, Monogr. Syst. Bot. Missouri Bot. Gard. 112: 120. 2007.

Pleurothallis mouraei Cogn. in Mart., Fl. Bras. 3(4): 580. 1896.

HABIT AND HABITAT: Epiphyte; riparian forest.

Voucher: Caetano s.n. (FURB 38759).

Pabstiella bradei (Schltr.) Luer, Monogr. Syst. Bot. Missouri Bot. Gard. 112: 119. 2007.*

Pleurothallis bradei Schltr., Anexos Mem. Inst. Butantan, Secç. Bot. 1(4): 41. 1922.

Trichosalpinx bradei (Schltr.) Luer, Phytologia 54(5): 394. 1983.

Specklinia bradei (Lindl.) Luer, Monogr. Syst. Bot. Missouri Bot. Gard. 95: 259. 2004.

HABIT AND HABITAT: Epiphyte; forest interior.

Voucher: Klein \& Bresolin 10883 (FLOR).

Pabstiella carinifera (Barb.Rodr.) Luer, Monogr. Syst. Bot. Missouri Bot. Gard. 112: 119. 2007.* [VU] Lepanthes carinifera Barb.Rodr., Rev. Engenh. 3(8): 110. 1881.

Pleurothallis carinifera (Barb.Rodr.) Cogn. in Mart., F1. Bras. 3(4): 584. 1896.

Trichosalpinx carinifera (Barb.Rodr.) Luer, Phytologia 54(5): 394. 1983.

Specklinia carinifera (Barb.Rodr.) Luer, Monogr. Syst. Bot. Missouri Bot. Gard. 95: 259. 2004.

HABIT AND HABITAT: Epiphyte; riparian forest, forest interior.

Vouchers: Schmitt et al. 1747 (FURB); Caetano s.n. (FURB 37332).

Pabstiella fusca (Lindl.) Chiron \& Xim.Bols., Richardiana 10(2): 56. 2010.*

Pleurothallis fusca Lindl., Companion Bot. Mag. 2: 354.1837.

Effusiella fusca (Lindl.) Campacci, Colet. Orquídeas Brasil. 9: 337. 2011.

Pleurothallis hypnicola Lindl., Edwards's Bot. Reg. 28(Misc.): 75. 1842.

Humboltia hypnicola (Lindl.) Kuntze, Revis. Gen. P1. 2: 667.1891.

Specklinia hypnicola (Lindl.) F.Barros, Hoehnea 10: 110. 1984.
Stelis hypnicola (Lindl.) Pridgeon \& M.W.Chase, Lindleyana 16(4): 263. 2001.

Pabstiella hypnicola (Lindl.) Luer, Monogr. Syst. Bot. Missouri Bot. Gard. 112: 120. 2007.

Effusiella hypnicola (Lindl.) Campacci, Colet. Orquídeas Brasil. 9: 338. 2011.

HABIT AND HABITAT: Epiphyte; riparian forest, forest interior.

Vouchers: Klein \& Bresolin 10884 (FLOR); Schmitt et al. 509 (FURB); Schmitt et al. 522 (FURB); Schmitt et al. 1775 (FURB); Schmitt et al. 1803 (FURB); Korte \& Kniess 988 (FURB); Caetano 184 (spirit, FURB).

Pabstiella matinhensis (Hoehne) Luer, Monogr. Syst. Bot. Missouri Bot. Gard. 112: 120. 2007.

Pleurothallis matinhensis Hoehne, Arq. Bot. Estado São Paulo, n.s. 1(1): 13. 1938.

Trichosalpinx matinhensis (Hoehne) Luer, Phytologia 54(5): 396. 1983.

Specklinia matinhensis (Hoehne) Luer, Monogr. Syst. Bot. Missouri Bot. Gard. 95: 262. 2004.

HABIT AND HABITAT: Epiphyte; riparian forest.

Voucher: Caetano s.n. (FURB 38877).

Pabstiella mirabilis (Schltr.) Brieger \& Senghas, Orchidee (Hamburg) 27(5): 195. 1976.*

Pleurothallis mirabilis Schltr., Notizbl. Bot. Gart. Berlin-Dahlem 7(66): 274. 1918.

Anthereon mirabilis (Schltr.) Pridgeon \& M.W.Chase, Lindleyana 16(4): 252. 2001.

Specklinia mirabilis (Schltr.) Luer, Monogr. Syst. Bot. Missouri Bot. Gard. 95: 262. 2004.

HABIT AND HABITAT: Epiphyte; riparian forest.

Voucher: Caetano s.n. (FURB 36258).

Pabstiella parvifolia (Lindl.) Luer, Monogr. Syst. Bot. Missouri Bot. Gard. 105: 139. 2006.

Pleurothallis parvifolia Lindl., Companion Bot. Mag. 2: 355. 1837.

Humboltia parvifolia (Lindl.) Kuntze, Revis. Gen. P1. 2: 668. 1891.

Specklinia parvifolia (Lindl.) Pridgeon \& M.W.Chase, Lindleyana 16(4): 258. 2001.

HABIT AND HABITAT: Epiphyte; forest interior. VOUCHER: Caetano 215 (spirit, FURB).

Pabstiella punctatifolia (Barb.Rodr.) Chiron, Phytotaxa 46: 55. 2012.*

Lepanthes punctatifolia Barb.Rodr., Gen. Spec. Orchid. 2: 55. 1881. 
Pleurothallis punctatifolia (Barb.Rodr.) Pabst, Orquídea (Rio de Janeiro) 28: 227. 1966.

Trichosalpinx punctatifolia (Barb.Rodr.) Luer, Phytologia 54(5): 397. 1983.

Specklinia punctatifolia (Barb.Rodr.) Luer, Monogr. Syst. Bot. Missouri Bot. Gard. 95: 263. 2004. HABIT AND HABITAT: Epiphyte; riparian forest.

VOUCHER: Caetano 58 (FURB).

Pabstiella rubrolineata (Hoehne) Luer, Monogr. Syst. Bot. Missouri Bot. Gard. 112: 120. 2007.*

Pleurothallis rubrolineata Hoehne, Bol. Agric. (São Paulo) 34: 609. 1934.

Specklinia rubrolineata (Hoehne) F.Barros, Hoehnea 10: 110. 1984.

HABIT AND HABITAT: Epiphyte; anthropic area (pasture).

Voucher: Caetano \& Almeida 208 (spirit, FURB).

Pabstiella seriata (Lindl.) Luer \& Toscano, Harvard Pap. Bot. 16(2): 381. 2011.

Pleurothallis seriata Lindl., Edwards's Bot. Reg. 26(Misc.): 75. 1840.

Humboltia seriata (Lindl.) Kuntze, Revis. Gen. Pl. 2: 668. 1891.

Specklinia seriata (Lind1.) Pridgeon \& M.W.Chase, Lindleyana 16(4): 259. 2001.

Panmorphia seriata (Lindl.) Luer, Monogr. Syst. Bot. Missouri Bot. Gard. 105: 174. 2006.

Anathallis seriata (Lindl.) Luer \& Toscano, Monogr. Syst. Bot. Missouri Bot. Gard. 115: 259. 2009.

Effusiella seriata (Lindl.) Baptista, Colet. Orquídeas Brasil. 9: 339. 2011.

HABIT AND HABITAT: Epiphyte; riparian forest.

VOUCHER: Caetano 219 (spirit, FURB).

Pabstiella stictophylla (Schltr.) J.Caetano \& L.R.S.Guim., comb. nov.*

Basionym: Pleurothallis stictophylla Schltr., Repert. Spec. Nov. Regni Veg. 23: 43. 1926; descr. emend. Hoehne, Bol. Agric. (São Paulo) 34: 608. 1934 ("1933”).

Trichosalpinx stictophylla (Schltr.) Luer, Phytologia 54(5): 397. 1983.

Specklinia stictophylla (Schltr.) Luer, Monogr. Syst. Bot. Missouri Bot. Gard. 95: 264. 2004.

Type: Brazil: Paraná, Porto de Cima, Hatschbach 89 (holotype $\mathrm{B} \dagger$ ). Lectotype designated here: original illustration of the flower analysis sketched

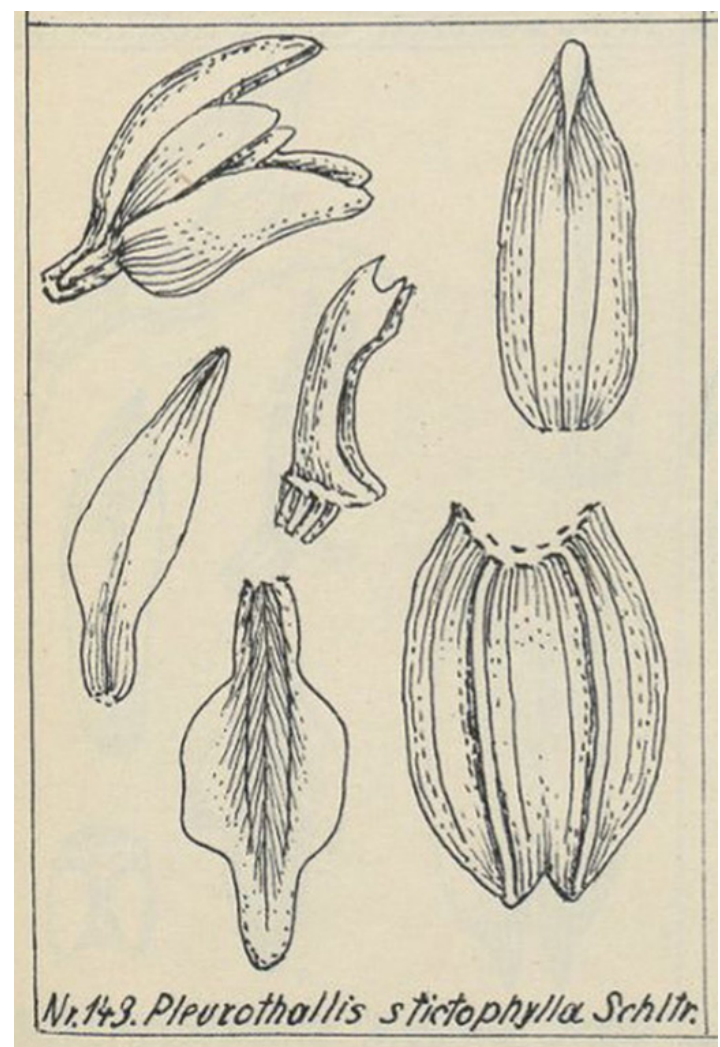

Figure 8. Pabstiella stictophylla. Lectotype of Pleurothallis stictophylla: original illustration in Mansfeld (1930: t. $\left.36, n^{\circ} .143\right)$.

by Schlechter and posthumously published by Mansfeld (1930: t. 36, nº 143!) (Fig. 8).

HABIT AND HABITAT: Epiphyte; riparian forest. VOUCHER: Bresolin \& Roco 696 (FLOR).

The description of the protologue, the emended description and the examination of the illustration of Pleurothallis stictophylla in Hoehne (1933) shows that this plant is clearly a species of Pabstiella, and thus this combination is needed. The study of many tropical orchids is severely hampered by the loss of all the holotypes of Schlechter's species due to the bombing of the Berlin Herbarium during the World War II (Merrill 1943, Butzin 1978, 1981, Hiepko 1987). Luckily, an original illustration in Mansfeld's work is available and can be used as taxonomic reference.

Pabstiella trifida (Lindl.) Luer, Monogr. Syst. Bot. Missouri Bot. Gard. 112: 120. 2007 (Fig. 9).

Pleurothallis trifida Lindl., Edwards's Bot. Reg. 28(Misc.): 82. 1842. 

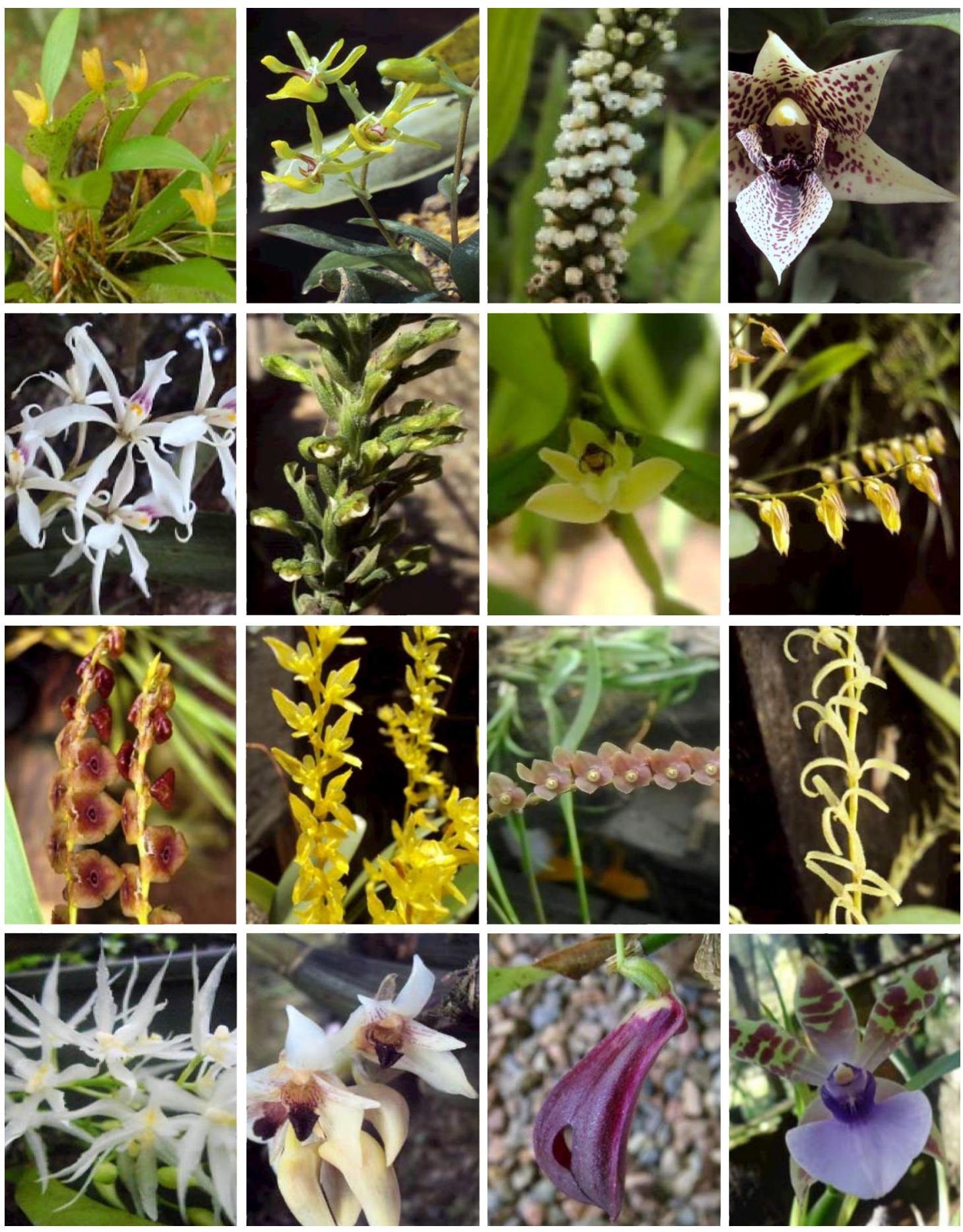

FIgURE 9. From top left to bottom right: Pabstiella trifida. Platyrhiza quadricolor. Prescottia densiflora. Promenaea rollissonii. Prosthechea glumacea. Sauroglossum elatum. Scaphyglottis modesta. Specklinia grobyi. Stelis megantha. S. montserratii. S. papaquerensis. S. sclerophylla. Warmingia eugenii. Xylobium variegatum. Zootrophion atropurpureum. Zygopetalum maxillare. All courtesy of The Field Museum, Chicago. 
Humboltia trifida (Lindl.) Kuntze, Revis. Gen. P1. 2: 668. 1891.

Specklinia trifida (Lindl.) F.Barros, Orchid Memories: 19. 2004.

HABIT AND HABITAT: Epiphyte; riparian forest, forest interior.

VOUCHERS: Schmitt et al. 516 (FURB); Caetano s.n. (FURB 38758).

Pabstiella uniflora (Lindl.) Luer, Monogr. Syst. Bot. Missouri Bot. Gard. 112: 121. 2007.

Pleurothallis uniflora Lindl., Companion Bot. Mag. 2(24): 355. 1837.

Humboltia uniflora (Lindl.) Kuntze, Revis. Gen. P1. 2: 668. 1891.

Specklinia uniflora (Lindl.) Pridgeon \& M.W.Chase, Lindleyana 16(4): 259. 2001.

Pleurothallis leontoglossa Rchb.f., Linnaea 22: 831. 1849.

Humboltia leontoglossa (Rchb.f.) Kuntze, Revis. Gen. Pl. 2: 667. 1891.

Specklinia leontoglossa (Rchb.f.) Luer, Monogr. Syst. Bot. Missouri Bot. Gard. 95: 261. 2004.

HABIT AND HABITAT: Epiphyte; riparian forest.

Voucher: Caetano 11 (FURB).

Pabstiella versicolor (Porsch) Luer, Monogr. Syst. Bot. Missouri Bot. Gard. 112: 121. 2007. [Toscano de Brito \& Luer 2016].*

Pleurothallis versicolor Porsch, Oesterr. Bot. Z. 55: 155. 1905.

Pleurothallis podoglossa Hoehne, Arq. Bot. Estado São Paulo, n.s. 1(1): 12. 1938.

Trichosalpinx podoglossa (Hoehne) Luer, Phytologia 54(5): 396. 1983.

Specklinia podoglossa (Hoehne) Luer, Monogr. Syst. Bot. Missouri Bot. Gard. 95: 263. 2004.

Pabstiella podoglossa (Hoehne) Luer, Monogr. Syst. Bot. Missouri Bot. Gard. 112: 120. 2007. Habit AND Habitat: Epiphyte; riparian forest. Voucher: Caetano 179 (spirit, FURB).

\section{Pelexia Poit. ex Lindl.}

Pelexia macropoda (Barb.Rodr.) Schltr., Beih. Bot. Centralbl. 37(2): 409. 1920.*

Spiranthes macropoda Barb.Rodr., Gen. Spec. Orchid. 1: 186. 1877.

Stenorrhynchos macropodum Barb.Rodr., Gen. Spec. Orchid. 1(Index): x. 1877.
HABIT AND HABITAT: Terrestrial; riparian forest. VOUCher: Caetano et al. 195 (spirit, FURB).

\section{Phymatidium Lindl.}

Phymatidium aquinoi Schltr., Repert. Spec. Nov. Regni Veg. Beih. 35: 101. 1925.*

HABIT AND HABITAT: Epiphyte; anthropic area (pasture). VOUCHER: Caetano 217 (spirit, FURB).

Phymatidium delicatulum Lindl., Gen. Sp. Orchid. P1.: 210. 1833 var. delicatulum.

Phymatidium myrtophilum Barb.Rodr., Gen. Spec. Orchid. 2: 229. 1882.

HABIT AND HABITAT: Epiphyte; riparian forest.

Vouchers: Caetano s.n. (FURB 37790); Caetano \& Lenz 62 (FURB).

\section{Platyrhiza Barb.Rodr.}

Platyrhiza quadricolor Barb.Rodr., Gen. Spec. Orchid. 2: 231.1882 (Fig. 9).*

HaBIT AND HABITAT: Epiphyte; riparian forest.

Voucher: Caetano s.n. (FURB 39735).

\section{Platystele Schltr}

Platystele oxyglossa (Schltr.) Garay, Orquideología 9(2): 120. 1974.

Pleurothallis oxyglossa Schltr., Repert. Spec. Nov. Regni Veg. 10: 354. 1912.

HABIT AND HABITAT: Epiphyte; riparian forest interior. Vouchers: Schmitt et al. 1812 (FURB); Caetano s.n. (FURB 38754).

\section{Polystachya Hook.}

Polystachya concreta (Jacq.) Garay \& H.R.Sweet, Orquideología 9(3): 206. 1974.

Epidendrum concretum Jacq., Enum. Syst. P1.: 30. 1760.

HABIT AND HABITAT: Epiphyte; anthropic area (pasture).

Voucher: Caetano s.n. (FURB 37806).

\section{Prescottia Lindl.}

Prescottia densiflora (Brongn.) Lindl., Ann. Mag. Nat. Hist. 6: 53.1840 (Fig. 9).*

Decaisnea densiflora Brongn., Voy. Monde, Phan.: 192. 1829.

Habit AND habitat: Terrestrial; forest edge.

Voucher: Caetano s.n. (FURB 39734). 
Prescottia stachyodes (Sw.) Lindl., Edwards's Bot. Reg. 22: t. 1915. 1836.

Cranichis stachyodes Sw., Prodr.: 120. 1788.

Prescottia colorans Lindl., Edwards's Bot. Reg. 22: t. 1915. 1836.

HABIT AND HABITAT: Terrestrial; forest interior, forest edge (top of hill).

Vouchers: Caetano 1 (FURB); Caetano 38 (FURB).

\section{Promenaea Lindl.}

Promenaea rollissonii (Lindl.) Lindl., Edwards's Bot. Reg. 29(Misc.): 13. 1843 (Fig. 9).*

Maxillaria rollissonii Lindl., Edwards's Bot. Reg. 23: t. 1986. 1837.

Zygopetalum rollissonii (Lindl.) Rchb.f. in Walp., Ann. Bot. Syst. 6(5): 659. 1863.

HABIT AND HABITAT: Epiphyte; riparian forest.

VOucher: Caetano 63 (FURB).

Prosthechea Knowles \& Westc.

Prosthechea bulbosa (Vell.) W.E.Higgins, Phytologia 82(5): 377.1998 ("1997”).

Epidendrum bulbosum Vell., Fl. Flumin. Icon. 9: t. 11.1831.

Encyclia bulbosa (Vell.) Pabst, Orquídea (Rio de Janeiro) 29(6): 276. 1972.

Anacheilium bulbosum (Vell.) Withner \& P.A.Harding, Cattleyas \& Relatives: Debatable Epidendrums: 54. 2004.

HABit AND HABITAT: Epiphyte; forest interior, anthropic area (pasture).

Vouchers: Korte \& Kniess 2110 (FURB); Caetano \& Schlemper 197 (spirit, FURB).

Prosthechea glumacea (Lindl.) W.E.Higgins, Phytologia 82(5): 378. 1998 ("1997") (Fig. 9).

Epidendrum glumaceum Lindl., Edwards's Bot. Reg. 25(Misc.): 38. 1839.

Hormidium glumaceum (Lindl.) Brieger, Publ. Ci. Inst. Genét. Esc. Super. Agric. Luiz de Queiroz 1: 19. 1960.

Encyclia glumacea (Lindl.) Pabst, Orquídea (Rio de Janeiro) 29(6): 276. 1972.

Anacheilium glumaceum (Lindl.) Pabst, Moutinho \& A.V.Pinto, Bradea 3(23): 183. 1981.

Epidendrum almasyi Hoehne, Arq. Bot. Estado São Paulo, n.s. 2: 84. 1947.
Hormidium almasyi (Lindl.) Brieger, Publ. Ci. Inst. Genét. Esc. Super. Agric. Luiz de Queiroz 1: 19. 1960. Encyclia almasii (Lindl.) Pabst, Orquídea (Rio de Janeiro) 29(6): 276. 1972.

HABIT AND HABITAT: Epiphyte; riparian forest.

VOUCHERs: Klein \& Bresolin 10888 (FLOR, ICN); Caetano s.n. (FURB 40571).

Prosthechea vespa (Vell.) W.E.Higgins, Phytologia 82(5): 381.1998 ("1997").*

Epidendrum vespa Vell., Fl. Flumin. Icon. 9: t. 27. 1831.

Encyclia vespa (Vell.) Dressler, Phytologia 21(7): 441. 1971.

Anacheilium vespa (Vell.) Pabst, Moutinho \& A.V.Pinto, Bradea 3(23): 184. 1981.

Habit AND HABITAT: Epiphyte; forest interior.

VOUCHER: Caetano 216 (spirit, FURB).

Psilochilus Barb.Rodr.

Psilochilus modestus Barb.Rodr., Gen. Spec. Orchid. 2: 273.1882.

Pogonia modesta (Barb.Rodr.) Cogn. in Mart., Fl. Bras. 3(4): 133. 1893.

HABIT AND HABITAT: Terrestrial; riparian forest.

Voucher: Caetano \& Almeida 223 (spirit, FURB).

Rodriguezia Ruiz \& Pav.

Rodriguezia bracteata (Vell.) Hoehne, Arq. Bot. Estado São Paulo, n.s. 2: 143. 1952.*

Epidendrum bracteatum Vell., Fl. Flumin. Icon. 9: t. 16.1831.

HABIT AND HABITAT: Epiphyte; anthropic area (pasture). Voucher: Caetano 209 (spirit, FURB).

Rodriguezia decora (Lem.) Rchb.f., Bot. Zeitung (Berlin) 10: 771. 1852.

Burlingtonia decora Lem., Jard. Fleur. 2(Misc.): 96. 1852.

HABIT AND HABITAT: Epiphyte; riparian forest.

Voucher: Caetano 15 (FURB).

\section{SACOILA Raf.}

Sacoila lanceolata (Aubl.) Garay, Bot. Mus. Leafl. 28(4): 352.9182 ("1980").

Limodorum lanceolatum Aubl., Hist. P1. Guiane 2: 821.1775.

Neottia lanceolata (Aubl.) Willd., Sp. Pl., ed. 4, 4(1): 73. 1805. 
Stenorrhynchos lanceolatum (Aubl.) Rich., De Orchid. Eur.: 37. 1817.

Spiranthes lanceolata (Aubl.) León, Contr. Ocas. Mus. Hist. Nat. Colegio "De La Salle" 8: 358. 1946.

Serapias coccinea Vell., Fl. Flumin. 9: t. 48. 1831. Stenorrhynchos coccineum (Vell.) Hoehne, Arq. Bot. Estado São Paulo, n.s. 2: 146. 1952.

Habit AND habitAT: Terrestrial; open area (forest regeneration).

Voucher: Caetano \& Schlemper 41 (FURB).

\section{Sauroglossum Lindl.}

Sauroglossum elatum Lindl., Edwards's Bot. Reg. 19: t. 1618. 1833 (Fig. 9).

HABIT AND HABITAT: Terrestrial; forest interior.

Voucher: Caetano s.n. (FURB 38761).

Scaphyglottis Poepp. \& Endl.

Scaphyglottis modesta (Rchb.f.) Schltr., Repert. Spec. Nov. Regni Veg. 23: 46. 1926 (Fig. 9).

Tetragamestus modestus Rchb.f., Bonplandia 2(2): 21. 1854.

Ponera modesta (Rchb.f.) Rchb.f., Linnaea 41: 85. 1876. HaBiT AND HABITAT: Epiphyte; riparian forest interior. VOUCHER: Caetano s.n. (FURB 36927).

SPeCKLINIA Lindl.

Specklinia grobyi (Bateman ex Lindl.) F.Barros, Hoehnea 10: 110. 1984 (Fig. 9).

Pleurothallis grobyi Bateman ex Lindl., Edwards's Bot. Reg. 21: t. 1797. 1835.

Humboltia grobyi (Bateman ex Lindl.) Kuntze, Revis. Gen. P1. 2: 667. 1891.

Habit AND HABITAT: Epiphyte; forest interior, riparian forest.

VOUCHERs: Bresolin 666 (CGMS, FLOR); Korte \& Kniess 2115 (FURB); Schmitt et al. 1743 (FURB); Caetano 178 (spirit, FURB).

\section{Stanhopea J.Frost ex Hook.}

Stanhopea lietzei (Regel) Schltr., Arch. Bot. São Paulo 1: 264. 1926.*

Stanhopea graveolens var. lietzei Regel, Gartenflora 40(8): 201. 1891.

HABIT AND HABITAT: Epiphyte; riparian forest.

Voucher: Caetano 191 (spirit, FURB).
Stelis Sw.

Stelis argentata Lindl., Edwards's Bot. Reg. 28(Misc.): 64. 1842.

Habit and habitat: Epiphyte; riparian forest.

Voucher: Caetano 180 (spirit, FURB).

Stelis ciliaris Lindl., Companion Bot. Mag. 2: 353.

1837. [Ignowski et al. 2015].

Apatostelis ciliaris (Lindl.) Garay, Bot. Mus. Leafl. 27(7-9): 188. 1979.

Stelis leinigii Pabst, Orquídea (Rio de Janeiro) 29:

7. 1967.

HaBIT AND HABITAT: Epiphyte; riparian forest.

Vouchers: Caetano s.n. (FURB 37339); Caetano 22 (FURB).

Stelis deregularis Barb.Rodr., Gen. Spec. Orchid. 2:

94. 1882.

Physosiphon deregularis (Barb.Rodr.) Cogn. in Mart., Fl. Bras. 3(4): 341. 1896.

Pseudostelis deregularis (Barb.Rodr.) Schltr., Anexos Mem. Inst. Butantan, Secç. Bot. 1(4): 38. 1922.

Pleurothallis deregularis (Barb.Rodr.) Luer, Selbyana 2(4): 385. 1978.

Physosiphon spiralis Lindl., Edwards's Bot. Reg. 21: t. 1797. 1835.

Pseudostelis spiralis (Lindl.) Schltr., Anexos Mem. Inst. Butantan, Secç. Bot. 1(4): 38. 1922.

HABIT AND HABITAT: Epiphyte; anthropic area (urban area).

Voucher: Caetano 13 (FURB).

Stelis intermedia Poepp. \& Endl., Nov. Gen. Sp. Pl. 1: 46.1836.

HABIT AND HABITAT: Epiphyte; forest interior.

VOUCHER: Schmitt et al. 1774 (FURB).

Stelis megantha Barb.Rodr., Gen. Spec. Orchid. 2: 83. 1881 (Fig. 9).*

Stelis macrochlamys Hoehne \& Schltr., Arch. Bot. São Paulo 1: 204. 1926.

HABIT AND HABITAT: Epiphyte; riparian forest.

Voucher: Caetano \& Henschel 40 (FURB).

Stelis montserratii (Porsch) Karremans, Lankesteriana 13(3): 329. 2014. [Karremans 2014] (Fig. 9).

Pleurothallis montserratii Porsch, Oesterr. Bot. Z. 55: 158. 1905.

Pleurothallis rubens Lindl., Edwards's Bot. Reg. 21: t. 1797. 1835.

Humboltia rubens (Lindl.) Kuntze, Revis. Gen. Pl. 2: 668. 1891. 
Specklinia rubens (Lindl.) F.Barros, Hoehnea 10: 110. 1984.

Anathallis rubens (Lind1.) Pridgeon \& M.W.Chase, Lindleyana 16(4): 250. 2001.

Stelis neorubens Chiron, Phytotaxa 46: 55. 2012.

HABIT AND HABITAT: Epiphyte; riparian forest.

VOUCHER: Caetano 186 (spirit, FURB).

Stelis papaquerensis Rchb.f., Linnaea 22: 822. 1850

(Fig. 9).

Stelis porschiana Schltr., Notizbl. Bot. Gart. Berlin-Dahlem 7: 270. 1917.

Stelis inaequisepala Hoehne \& Schltr., Anexos Mem. Inst. Butantan, Secç. Bot. 1(2): 30. 1921.

Stelis juergensii Schltr., Repert. Spec. Nov. Regni Veg. Beih. 35: 49. 1925.

HABIT AND HABITAT: Epiphyte; riparian forest.

Vouchers: Bresolin 662 (FLOR); Caetano 34 (FURB).

Stelis sclerophylla (Lindl.) Karremans, Lankesteriana 13(3): 330. 2014 (Fig. 9).

Pleurothallis sclerophylla Lindl., Edwards's Bot. Reg. 21: t. 1797. 1835.

Humboltia sclerophylla (Lindl.) Kuntze, Revis. Gen. P1. 2: 668. 1891.

Anathallis sclerophylla (Lindl.) Pridgeon \& M.W.Chase, Lindleyana 16(4): 250. 2001.

Specklinia sclerophylla (Lindl.) Luer, Monogr. Syst. Bot. Missouri Bot. Gard. 95: 263. 2004.

HABIT AND HABITAT: Epiphyte; riparian forest.

VOUCher: Caetano 66 (FURB).

Trichocentrum Poepp. \& Endl.

Trichocentrum pumilum (Lindl.) M.W.Chase \& N.H.Williams, Lindleyana 16(2): 138. 2001.

Oncidium pumilum Lindl., Bot. Reg. 11: t. 920.1825. Lophiaris pumila (Lindl.) Braem, Schlechteriana 4(1-2): 21. 1993.

Lophiarella pumila (Lindl.) Szlach., Mytnik \& Romowicz, Polish Bot. J. 51(1): 54. 2006.

HABIT AND HABITAT: Epiphyte; anthropic area (pasture). VOUCHER: Caetano 182 (spirit, FURB).

\section{Trichosalpinx Luer}

Trichosalpinx montana (Barb.Rodr.) Luer, Phytologia 54(5): 396. 1983.*

Lepanthes montana Barb.Rodr., Gen. Spec. Orchid. 1: 22. 1877.
Pleurothallis lepanthipoda Hoehne \& Schltr., Arch. Bot. São Paulo 1: 218. 1926.

HaBiT AND HABITAT: Epiphyte; forest interior. VOUCher: Caetano 214 (spirit, FURB).

TrizeuXIs Lindl.

Trizeuxis falcata Lindl., Coll. Bot.: t. 2. 1821.

HABIT AND HABITAT: Epiphyte; forest interior, forest edge.

Vouchers: Caetano 4 (FURB); Caetano s.n. (FURB 36630).

\section{WARMingIa Rchb.f.}

Warmingia eugenii Rchb.f., Otia Bot. Hamburg.: 87. 1881 (Fig. 9).

HaBiT AND HABITAT: Epiphyte; riparian forest.

VOUCHER: Caetano 14 (FURB).

\section{XуLовıum Lindl.}

Xylobium variegatum (Ruiz \& Pav.) Garay \& Dunst., Venez. Orchids Ill. 2: 342. 1961 (Fig. 9).

Maxillaria variegata Ruiz \& Pav., Syst. Veg. Fl. Peruv. Chil. 1: 222. 1798.

Dendrobium variegatum (Ruiz \& Pav.) Pers., Syn. Pl. 2(2): 524. 1807.

HABIT AND HABITAT: Epiphyte; riparian forest.

Voucher: Caetano 79 (FURB).

\section{Zootrophion Luer}

Zootrophion atropurpureum (Lindl.) Luer, Selbyana 7(1): 80.1982 (Fig. 9).

Specklinia atropurpurea Lindl., Edwards's Bot. Reg. 21: t. 1797. 1835.

Pleurothallis atropurpurea (Lindl.) Lindl., Edwards's Bot. Reg. 28(Misc.): 81. 1842.

Cryptophoranthus atropurpureus (Lindl.) Rolfe, Gard. Chron., ser. 3, 2: 693. 1887.

Humboltia atropurpurea (Lindl.) Kuntze, Revis. Gen. P1. 2: 667. 1891.

HABIT AND HABITAT: Epiphyte; riparian forest.

VOUCHER: Caetano s.n. (FURB 37960).

\section{Zygopetalum Hook.}

Zygopetalum crinitum G.Lodd., Bot. Cab. 17: t. 1687. 1831.*

HABIT AND HABITAT: Epiphyte; forest interior. VouCHERs: Schmitt et al. 526 (FURB, UPCB). 
Zygopetalum maxillare G.Lodd., Bot. Cab. 18: t. 1776. 1832 (Fig. 9).

HABit AND hABITAT: Epiphyte; forest interior (riparian forest).

VOucher: Caetano \& Schlemper 24 (FURB).
Zygostates Lindl.

Zygostates pellucida Rchb.f. in Walp., Ann. Bot. Syst. 6(4): 564. 1863.*

HaBiT AND HABITAT: Epiphyte; riparian forest.

Voucher: Caetano s.n. (FURB 37914).

AcKnowledgments. We thank Dr. Fabio de Barros (IBt) for the identification of some species, Dr Eric C. Smidt (UFPR) for taxonomic information on Stelis, and Dr Climbiê F. Hall (MPEG) for suggestions and review the English text. LRSG thanks the São Paulo Research Foundation (FAPESP, process 2010/16353-1) for the assistance in the field incursions.

\section{LiTERATURE CITED}

Abreu, N. L., Menini Neto, L. \& Kuono, T. U. P. (2011). Orchidaceae das Serras Negra e do Funil, Rio Preto, Minas Gerais, e similaridade florística entre formações campestres e florestais do Brasil. Acta Botanica Brasilica, 25(1), 58-70. doi: http://dx.doi.org/10.1590/ S0102-33062011000100009

Alvares, C. A., Stape, J. L., Sentelhas, P. C., Gonçalves, J. L. M. \& Sparovek, G. (2013). Köppen's climate classification map for Brazil. Meteorologische Zeitschrift, 22(6), 711-728. doi: http://dx.doi org/10.1127/0941-2948/2013/0507

Barros, F. (1983). Flora fanerogâmica da reserva do Parque Estadual das Fontes do Ipiranga (São Paulo, Brasil.). 198 - Orchidaceae. Hoehnea, 10, 74-124.

BFG (The Brazil Flora Group). (2015). Growing knowledge: an overview of Seed Plant diversity in Brazil. Rodriguésia, 66(4), 1085-1113. doi: http:// dx.doi.org/10.1590/2175-7860201566411

Butzin, F. (1978). In Berlin vorhandene Typen von Schlechters Orchideenarten. Willdenowia, 8, 401-407.

Butzin, F. (1981). Typenstudien im Berliner OrchideenHerbar: Diverse markierte Typen. Willdenowia, 11, 119-121.

Caetano, J. O. \& Guimarães, L. R. S. (2013). A família Orchidaceae no município de Benedito Novo, Santa Catarina. Boletim CAOB, 89, 3-11.

Caetano, J. O., Schlemper, C. R., Henschel, S. \& Guimarães, L. R. S. (2013). A família Orchidaceae no município de Benedito Novo, Santa Catarina II: Adições e correções. Boletim CAOB, 91, 90-95.

Catharino, E. L. M. \& Barros, F. (2004). Orquídeas do Maciço da Juréia e arredores. In: O. A. V. Marques \& W. Duleba (Eds.). Estação Ecológica Juréia-Itatins: ambiente físico, flora e fauna (pp. 152-161). Ribeirão Preto, SP: Holos.

Chase, M. W., Cameron, K. M., Freudenstein, J. V., Pridgeon, A. M., Salazar, G., van den Berg. C. \& Schuiteman, A. (2015). An updated classification of Orchidaceae. Botanical Journal of the Linnean Society, 177(2), 151-
174. doi: http://dx.doi.org/10.1111/boj.12234

Climate-Data.org (2016). Climate: Benedito Novo. In: Climate-Data.org / AmbiWeb GmbH. Gernsbach. Recovered from http://en.climate-data.org/ location/43739/ (accessed 19 August 2016).

Favretto, M. A. \& Geuster, C. J. (2011). Orquideas e bromélias do Vale do Rio do Peixe, Santa Catarina. Joaçaba, SC: Clube de Autores Publicações S/A.

Filgueiras, T. S., Nogueira, P. E., Brochado, A. L. \& Guala II, G. F. (1994). Caminhamento: um método expedito para levantamentos florísticos qualitativos. Cadernos de Geociências, 12, 39-43.

Flora do Brasil 2020 (2016). Rio de Janeiro: Jardim Botânico do Rio de Janeiro. Recovered from http://floradobrasil.jbrj. gov.br/ (accessed 11 October 2016).

Fraga, C. N. \& Peixoto, A. L. (2004). Florística e ecologia das Orchidaceae das restingas do estado do Espírito Santo. Rodriguésia, 55(84), 5-20.

Fundação SOS Mata Atlântica \& INPE. (2011). Atlas dos remanescentes florestais da Mata Atlântica período 2008-2010. São Paulo: Fundação SOS Mata Atlântica/ Instituto Nacional de Pesquisas Espaciais.

Govaerts, R., Bernet, P., Kratochvil, K., Gerlach, G., Carr, G., Alrich, P., Pridgeon, A. M., Pfahl, J., Campacci, M. A., Baptista, D. H., Tigges, H., Shaw, J., Cribb, P. J., George, A., Kreuz, K. \& Wood, J. (2015). World Checklist of Orchidaceae. Kew: Royal Botanic Gardens. Recuperado de http://apps.kew.org/wcsp/ (accessed 14 August 2016).

Guimarães, L. R. S., Caetano, J. O., Schlemper, C. R., Couto, C. C. M. \& Nascimento, M. V. (2016). Novelties in Orchidaceae from the State of Santa Catarina, Brazil. Richardiana, 16, 249-257.

Hatschbach, G. G. \& Ziller, S. R. (1995). Lista vermelha de plantas ameaçadas de extinção no estado do Paraná. Curitiba: SEMA/GTZ.

Hiepko, P. (1987). The collections of the Botanical Museum Berlin-Dahlem (B) and their history. Englera, 7, 219252. 
Hoehne, F. C. (1933). Contribuições para o conhecimento da flora orchidologica brasilica III. Boletim de Agricultura (São Paulo), 34, 600-638.

Ignowski, H., Toscano de Brito, A. L. V., Bona, C. \& Smidt, E. C. (2015). Nomenclatural notes on Stelis ciliaris (Pleurothallidinae, Orchidaceae). Phytotaxa, 218(1), 30-38. doi: http://dx.doi.org/10.11646/ phytotaxa.218.1.2

Karremans, A. P. (2014). Lankesteriana, a new genus in the Pleurothallidinae (Orchidaceae). Lankesteriana, 13(3), 319-332. http://dx.doi.org/10.15517/lank.v13i3.14368

Klein, R. M. (1979). Ecologia da flora e vegetação do Vale do Itajaí. Sellowia, 31(1), 1-164.

Klein, R. M., Bresolin, A. \& Reis, A. (1977/1978). Distribuição de orquídeas da Ilha de Santa Catarina e arredores. Insula, 9(1), 4-29.

Kollmann, L. J. C., Fontana, A. P., Simonelli, M. \& Fraga, C. N. (2007). As Angiospermas ameaçadas de extinção no Estado do Espírito Santo. In: M. Simonelli \& C. N. Fraga (Orgs). Espécies da flora ameaçadas de extinção no Estado do Espírito Santo (pp. 105-137). Vitória: Ipema.

Köppen, W. (1936). Das geographische System der Klimate. In: W. R. Köppen \& R. Geiger (Eds.). Handbuch der Klimatologie. Volume I, Part C (pp. 1-44). Berlin: Gebrüder Bornträger.

Lenzi, M., Matos, J. Z., Fraga, A. M. \& Crespo, M. B. (2015). Orchids of the State Park of Serra do Tabuleiro, Southern Brazil. Anales del Jardín Botánico de Madrid, 72(2), e020. doi: http://dx.doi.org/10.3989/ajbm.2345

Leoni, L. S. (1992). Lista das plantas vasculares do Parque Estadual da Serra do Brigadeiro, Minas Gerais. Pabstia, $3,1-13$.

Leoni, L. S. (1997). Catálogo preliminar das fanerógamas ocorrentes no Parque Nacional do Caparaó - MG. Pabstia, 8(2), 1-28.

Luer, C. A. \& Toscano de Brito, A. L. V. (2011). Miscellaneous new species and combinations in the Pleurothallidinae (Orchidaceae) from Brazil and Argentina. Harvard Papers in Botany, 16(2), 361-382. doi: http://dx.doi.org/10.3100/0.25.016.0207

Mamede, M. C. H., Souza, V. C., Prado, J., Barros, F., Wanderley, M. G. L. \& Rando, J. G. (Orgs.). (2007). Livro vermelho das espécies vegetais ameaçadas do Estado de São Paulo. São Paulo: Instituto de Botânica.

Mansfeld, R. (1930). Blütenanalysen neuer Orchideen von R. Schlechter (†). I. Südamerikanische Orchideen. Repertorium specierum novarum regni vegetabilis; Beihefte, 58, 1-7, t. 1-60.

Menini Neto, L., Almeida, V. R. \& Forzza, R. C. (2004). A família Orchidaceae na Reserva Biológica da Represa do Grama - Descoberto, Minas Gerais, Brasil. Rodriguésia, 55(84), 137-156.
Menini Neto, L., Barros, F., Vinhos, F., Furtado, S. G., Judice, D. M., Fernandez, E. P., Sfair, J. C., Barros, F. S. M., Prieto, P. V., Kutschenko, D. C., Moraes, M. A., Zanata, M. R. V. \& Santos Filho, L. A. F. (2013). Orchidaceae. In: G. Martinelli \& M. A. Moraes (Eds.). Livro vermelho da flora do Brasil (pp. 749-818). Rio de Janeiro: Instituto de Pesquisas Jardim Botânico do Rio de Janeiro.

Merrill, E. D. (1943). Destruction of the Berlin Herbarium. Science, 3, 490-491. doi: http://dx.doi.org/10.1126/ science.98.2553.490

Mittermeier, R. A., Gil, P. G., Hoffmann, M., Pilgrim, J., Brooks, T., Mittermeier, C. G., Lamoreux, J. \& Fonseca, G. A. B. (2004). Hotspots Revisited: Earth's biologically richest and most endangered terrestrial ecoregions. Mexico City: CEMEX.

Mori, S. A., Mattos-Silva, L. A., Lisboa, G. \& Coradin, L. (1985). Manual de Manejo do Herbário Fanerogâmico. Ilhéus, BA: CEPLAC/CEPEC.

Myers, N., Mittermeier, R. A., Mittermeier, C. G., Fonseca, G. A. B. \& Kent, J. (2000). Biodiversity hotspots for conservation priorities. Nature, 403, 853-858. doi: http://dx.doi.org/10.1038/35002501

Pabst, G. F. J. (1951). Contribuição para o conhecimento das orquídeas de Santa Catarina e sua dispersão geográfica I. Anais Botânicos do Herbário "Barbosa Rodrigues", 3, 41-53.

Pabst, G. F. J. (1952). Contribuição para o conhecimento das orquídeas de Santa Catarina e sua dispersão geográfica I (continuação). Anais Botânicos do Herbário "Barbosa Rodrigues", 4, 69-86.

Pabst, G. F. J. (1953). Contribuição para o conhecimento das orquídeas de Santa Catarina e sua dispersão geográfica I (conclusão). Anais Botânicos do Herbário "Barbosa Rodrigues", 5, 39-93.

Pabst, G. F. J. (1954). Contribuição para o conhecimento das orquídeas de Santa Catarina e sua dispersão geográfica II. Sellowia, 6, 181-197.

Pabst, G. F. J. (1956). Contribuição para o conhecimento das orquídeas de Santa Catarina e sua dispersão geográfica III. Sellowia, 7/8, 175-181.

Pabst, G. F. J. (1957). Contribuição para o conhecimento das orquídeas de Santa Catarina e sua dispersão geográfica IV. Sellowia, 9, 249-256.

Pabst, G. F. J. (1959). Contribuição para o conhecimento das orquídeas de Santa Catarina e sua dispersão geográfica V. Sellowia, 10, 163-166.

Pansarin, E. R. \& Pansarin, L. M. (2008). A família Orchidaceae na Serra do Japi, São Paulo, Brasil. Rodriguésia, 59(1), 99-111.

Pessoa, E. \& Alves, M. (2015). Synopsis of Orchidaceae from Serra do Urubu: an area of montane forest, Pernambuco State, Brazil. Hoehnea, 42(1), 109-133. 
doi: http://dx.doi.org/10.1590/2236-8906-35/2014

Ranta, P., Blom, T., Niemelä, J., Joensuu, E. \& Siitonen, M. (1998). The fragmented Atlantic rain forest of Brazil: size, shape and distribution of forest fragments. Biodiversity and Conservation, 7(3), 385-403. doi: http://dx.doi.org/10.1023/A:1008885813543

Reis, A., Boeira, A. F., Dametto, A., Sanches, A. C., Souza, A. C., Neto, A. A. C., Neves, B. T., Longo, B. L., Barcellos, C. S., Perart, D., Freitas, D. M., Zech, D. F., Matos, E. P. M., Pansera, E. G., Marchioretto, L., Bileski, M. K. S., Machado, M. S. C., Heinz, M. K., Cury, R. K., Spuldaro, S. C., Quevedo, T. C., Zanotto, T. L. \& Soboleski, V. F. (2011). Listas das espécies vegetais catarinenses da divisão Angiospermas. Sellowia, 56(63), 11-256.

Ribeiro, M. C., Metzger, J. P., Martensen, A. C., Ponzoni, F. J. \& Hirota, M. M. (2009). The Brazilian Atlantic Forest: How much is left, and how is the remaining forest distributed? Implications for conservation. Biological Conservation, 142(6), 1144-1153. doi: http://dx.doi.org/10.1016/j.biocon.2009.02.021

Rio Grande do Sul. (2002). Decreto estadual CONSEMA $n^{\circ}$ 42.099. Declara as espécies da flora nativa ameaçadas de extinção no estado do Rio Grande do Sul e da outras providências. Conselho Estadual do Meio Ambiente. Palácio Piratini, Porto Alegre. 31 Dezembro 2002.

Rodrigues, V. T. \& Barros, F. (2012). A família Orchidaceae da Serra do Itapeti. In: M. S. C. Morini \& V. F. O. Miranda (Orgs.). Serra do Itapeti: Aspectos históricos, sociais e naturalisticos (pp. 127-141). Bauru, SP: Canal 6.

Rohr, J. A. (1951). Orquídeas. Relatório do Colégio Catarinense, s.n., 5-18.

Romanini, R. P. \& Barros, F. (2007). Orchidaceae. In: M. M. R. F. Melo, F. Barros, S. A. C. Chiea, M. Kirizawa, S. L. Jung-Mendaçolli \& M. G. L. Wanderley (Eds.). Flora Fanerogâmica da Ilha do Cardoso, Volume 12 (pp. 29-275). São Paulo: Instituto de Botânica.
Siqueira, C. E., Zanin, A. \& Menini Neto, L. (2014). Orchidaceae in Santa Catarina: update, geographic distribution and conservation. Check List, 10(6), 14521478. doi: http://dx.doi.org/10.15560/10.6.1452

Stehmann, J. R., Forzza, R.C., Sobral, M. \& Kamino, L. H. Y. (2009). Gimnospermas e Angiospermas. In: J. R. Stehmann, R. C. Forzza, A. Salino, M. Sobral, D. P. Costa \& L. H. Y. Kamino (Eds.). Plantas da Floresta Atlântica (pp. 27-37). Rio de Janeiro: Jardim Botânico do Rio de Janeiro.

Thiers, B. (2016). Index Herbariorum: A global directory of public herbaria and associated staff. New York Botanical Garden's Virtual Herbarium. Recovered from http://sweetgum.nybg.org/ih/ (accessed 7 July 2016).

Toscano de Brito, A. L. V. \& Luer, C. A. (2015). New species and nomenclatural notes in the Pleurothallidinae (Orchidaceae) from Brazil. Harvard Papers in Botany, 20(1), 39-68. doi: http://dx.doi.org/10.3100/hpib. v20iss 1.2015.n4

Toscano de Brito, A. L. V. \& Luer, C. A. (2016). New species and nomenclatural notes in Pabstiella (Orchidaceae: Pleurothallidinae) from Brazil. Lankesteriana, 16(2), 153-185. doi: http://dx.doi.org/10.15517/lank. v16i2.25997

Vibrans, A. C., McRoberts, R. E., Lingner, D. V., Nicoletti, A. L. \& Moser, P. (2012). Extensão original e atual da cobertura florestal de Santa Catarina. In: A. C. Vibrans, L. Sevegnani, A. L. Gasper \& D. V. Lingner (Eds.). Inventário Florístico Florestal de Santa Catarina. Volume 1: Diversidade e conservação dos remanescentes florestais (pp. 65-76). Blumenau, SC: Edifurb.

Zandoná, L. R. \& Catharino, E. L. M. (2015). Orchidaceae no Parque Estadual da Cantareira e sua conservação. Revista do Instituto Florestal, 27(1), 83-101. doi: http:// dx.doi.org/10.4322/rif.2015.007 